\title{
A framework for Higgs characterisation
}

\author{
P. Artoisenet, ${ }^{a}$ P. de Aquino, ${ }^{b}$ F. Demartin, ${ }^{c}$ R. Frederix, ${ }^{d}$ S. Frixione, ${ }^{d, e}$ F. Maltoni, ${ }^{c}$ \\ M. K. Mandal, ${ }^{f}$ P. Mathews, ${ }^{g}$ K. Mawatari, ${ }^{b}$ V. Ravindran, ${ }^{h}$ S. Seth, ${ }^{g}$ P. Torrielli ${ }^{i}$ \\ and M. Zaro ${ }^{c}$
}

${ }^{a}$ Nikhef Theory Group, Science Park 105, 1098 XG Amsterdam, The Netherlands

${ }^{b}$ Theoretische Natuurkunde and IIHE/ELEM, Vrije Universiteit Brussel, and International Solvay Institutes,

Pleinlaan 2, B-1050 Brussels, Belgium

${ }^{c}$ Centre for Cosmology, Particle Physics and Phenomenology (CP3),

Université Catholique de Louvain,

B-1348 Louvain-la-Neuve, Belgium

${ }^{d}$ PH Department, TH Unit, CERN,

CH-1211 Geneva 23, Switzerland

e ITPP, École Polytechnique Fédérale de Lausanne (EPFL),

CH-1015 Lausanne, Switzerland

${ }^{f}$ Regional Centre for Accelerator-based Particle Physics, Harish-Chandra Research Institute, Chhatnag Road, Jhunsi, Allahabad 211019, India

${ }^{g}$ Saha Institute of Nuclear Physics, 1/AF Bidhan Nagar, Kolkata 700 064, India

${ }^{h}$ Institute of Mathematical Sciences, CIT Campus, Taramani, Chennai-113, India

${ }^{i}$ Institut für Theoretische Physik, Universität Zürich,

Winterthurerstrasse 190, CH-8057 Zürich, Switzerland

E-mail: partois@nikhef.nl, paquino@vub.ac.be,

federico.demartin@uclouvain.be, rikkert.frederix@cern.ch,

frixione@mail.cern.ch, fabio.maltoni@uclouvain.be, mandal@hri.res.in,

prakash.mathews@saha.ac.in, kentarou.mawatari@vub.ac.be,

ravindra@imsc.res.in, satyajit.seth@saha.ac.in,

torriell@physik.uzh.ch, marco.zaro@uclouvain.be 
ABStRact: We introduce a framework, based on an effective field theory approach, that allows one to perform characterisation studies of the boson recently discovered at the LHC, for all the relevant channels and in a consistent, systematic and accurate way. The production and decay of such a boson with various spin and parity assignments can be simulated by means of multi-parton, tree-level matrix elements and of next-to-leading order QCD calculations, both matched with parton showers. Several sample applications are presented which show, in particular, that beyond-leading-order effects in QCD have nontrivial phenomenological implications.

Keywords: Phenomenological Models, Monte Carlo Simulations

ARXIV EPRINT: 1306.6464 


\section{Contents}

1 Introduction 1

2 Effective lagrangian $\quad 3$

2.1 Spin $0 \quad 4$

2.2 Spin $1 \quad 6$

2.3 Spin $2 \quad 8$

3 Validation and comparisons $\quad 8$

$\begin{array}{ll}3.1 \text { Leading-order parton-level results } & 9\end{array}$

$\begin{array}{ll}3.2 & \text { Higher orders in QCD } \\ & 12\end{array}$

3.2.1 Inclusive production $p p \rightarrow X\left(J^{P}\right): \mathrm{ME}+\mathrm{PS}$ vs. aMC@NLO 13

4 Applications $\quad 17$

4.1 Unitarity-violating behaviour of models with a spin-2 state 17

$\begin{array}{lll}4.2 & \text { Higher order QCD effects on spin observables for a spin-2 state } & 19\end{array}$

4.3 Determination of the $C P$-mixing of a spin- 0 state with the matrix element $\begin{array}{ll}\text { method } & 21\end{array}$

5 Summary and outlook $\quad 25$

$\begin{array}{ll}\text { A Spin-1 hypothesis and two-photon final states } & 26\end{array}$

B Divergences in the $p p \rightarrow X_{2}$ computation at NLO 29

\section{Introduction}

Any major discovery is the beginning of a new journey. With the luminosity accumulated by the LHC the existence of a new boson with a mass of about $125 \mathrm{GeV}$ has by now reached an overwhelming evidence $[1,2]$. In addition, several independent, yet preliminary, studies [3-10] give rather strong indications that the new particle is indeed a parity-even scalar, with the properties predicted by the Standard Model (SM) [11]. Were this the case, we would have the first evidence for the actual relevance of the Brout-Englert-Higgs mechanism $[12,13]$, together with the discovery of the first elementary scalar particle. Furthermore, and maybe even more far reaching, this would mean that a genuinely different short-range interaction of Yukawa type, i.e. not under the spell of a gauge principle, is at work in the Universe.

Assessing beyond any reasonable doubt that the new boson $i s$ the scalar particle predicted by the SM is therefore an endeavour of utmost importance. 
The questions to be addressed can be organised into two levels, assuming that only one resonance has been observed. At the lowest level (let us call it Level 1) there are the questions on the very nature of the new particle, such as what are its spin and parity. At the next level (Level 2) one must investigate the interactions of such a resonance with SM particles. Several approaches have been proposed to address questions at both levels, with different degrees of observable/model dependence and generality.

The first possibility is that of focussing on specific processes and observables, and of analysing their sensitivity to a given hypothesis, such as the spin or the parity of the resonance. This approach has the advantage that it can give useful indications to experimental analyses on the most sensitive observables and, in some cases, can be made really model independent (see for instance ref. [14]). On the other hand, it is normally limited to predictions at the lowest order, and by construction it does not provide a framework where all information regarding the resonance can be collected and globally analysed.

A more general approach is that of writing all vertices which enter a given set of processes (as for example the three-particle vertices in $p p \rightarrow X\left(J^{P}\right) \rightarrow V V, f \bar{f}$ with $V=$ $\gamma, W, Z$ ) in the most general form compatible with the desired symmetries (which implies non-SM, i.e. anomalous, couplings [15-17]). A positive aspect of this approach is that it gives the possibility to promote couplings to form factors, since no assumptions are made on where new physics might lie. Such form factors also allow one to reinstate unitarity in case of need, at the price of introducing an explicit model dependence. As a possible shortcoming of this approach, higher orders in QCD and electroweak (EW) couplings are, in general, more difficult to include. Finally, a plethora of new parameters are needed without the possibility of establishing any hierarchy among them.

A third very common and powerful approach is that of relying on an effective field theory valid up to a scale $\Lambda$, that features only one new state $X\left(J^{P}\right)$ at the EW scale $v$; furthermore, one assumes that any other new (i.e., non-SM) state resides at scales larger than or equal to $\Lambda$. One can show that a theory of this kind is renormalizable order by order in the $\sqrt{\hat{s}} / \Lambda$ expansion, that it can be systematically improved by adding operators of higher dimensions and $\mathrm{QCD} / \mathrm{EW}$ corrections, and that in general it depends only on a few free parameters, since the gauge symmetries and the hierarchy in terms of the number of canonical dimensions of the relevant operators drastically reduce the number of allowed terms. The drawback of any effective field theory is that by construction it assumes no new physics below $\Lambda$, and that it violates unitarity and loses predictivity for $\sqrt{\hat{s}}>\Lambda$; still, it remains an exceedingly viable approach to the problem of new-particle characterisation, in particular to Level-2 questions, and as such it has been widely advocated in the context of the Higgs discovery (see for example refs. [18-38], and more in general refs. [39, 40]).

The goal of this work is that of presenting the implementation of a simple effectivefield lagrangian below the electroweak symmetry breaking (EWSB) scale, devised with Level-1 issues in mind, yet perfectly suitable to also address questions on the strength of the Higgs couplings. The framework we propose is minimal, yet it has the advantage of being systematically improvable through the inclusion of higher-order corrections, notably those coming from QCD; in the following, we shall amply exploit this feature, and the 
opportunities for accurate simulations it provides, in the context both of multi-parton tree-level (MADGRAPH 5) and of next-to-leading order (AMC@NLO) computations.

In a nutshell, our assumptions are simply that the resonance structure observed in data corresponds to one bosonic state $\left(X\left(J^{P}\right)\right.$ with $J=0,1$, or 2 , and a mass of about $125 \mathrm{GeV}$ ), and that no other new state below the cutoff $\Lambda$ coupled to such a resonance exists. We also follow the principle that any new physics is dominantly described by the lowest dimensional operators. This means, for example, that for the spin- $0 C P$-even case (which corresponds to the SM scalar) we include all effects coming from the set of dimension-six operators relevant to the Higgs three-point couplings. ${ }^{1}$ Given that our goal is that of providing a simulation framework in terms of mass eigenstates, and consistently with the general guidelines outlined above, we construct an effective lagrangian below the EWSB scale, where $\mathrm{SU}(2)_{L} \times \mathrm{U}(1)_{Y}$ reduces to $\mathrm{U}(1)_{E M}$; moreover, we do not require $C P$ conservation, and we leave open the possibility that the new boson might be a scalar with no definite $C P$ properties.

The paper is organised as follows. In section 2 we introduce the effective lagrangians for spin 0,1 , and 2 which form the basis of this work, and discuss in detail their characteristics. In section 3 we deal with the implementation of these lagrangians in FEYNRULES and MADGRAPH 5 and its subsequent validation, and with the capabilities of the resulting framework for simulations that emphasise accuracy, namely AMC@NLO and tree-level matrix element/parton shower merging (ME+PS). In section 4 we turn to give a few sample applications: the high-energy behaviour of a spin-2 hypothesis with non-universal couplings to SM particles, the effects of initial-state QCD radiation on spin-correlation observables, and an application of the matrix element method to the determination of the amount of $C P$-mixing of a spin-0 resonance. In section 5 we present our conclusions and give a brief outlook on future prospects. Some technical details are collected in the appendices.

\section{Effective lagrangian}

Our effective field theory consists of the SM (except for the Higgs itself), expressed through the physical degrees of freedom present below the EWSB scale, plus a new bosonic state $X\left(J^{P}\right)$ with spin/parity assignments $J^{P}=0^{+}, 0^{-}, 1^{+}, 1^{-}$, and $2^{+}$. The new state can couple to SM particles via interactions of the lowest possible dimensions. In addition, the state $0^{+}$is allowed to mix with the $0^{-}$one, and can interact with SM particles with higher-dimensional operators beyond those of the SM. Technically, the implementation of the lagrangian is performed following the path outlined in ref. [41], i.e., starting from FEynRules [42] by extending and completing an earlier version of the model used in ref. [43]. The model particle content and its Feynman rules can be exported to any matrix element generator in the UFO [44]. We dub it Higgs Characterisation model; it is publicly available at the FEYnRules on-line database [45].

\footnotetext{
${ }^{1}$ The extension of our effective Lagrangian to include operators generating new four-point interactions is straightforward.
} 
The lagrangian of our model relevant to the physics of the boson $X\left(J^{P}\right)$ is written as follows:

$$
\mathcal{L}_{H C, J}=\mathcal{L}_{S M-H}+\mathcal{L}_{J}
$$

where the first term on the r.h.s. describes the SM degrees of freedom except for the Higgs, and $\mathcal{L}_{J}$ contains the kinetic and interaction terms (with SM particles) of the new bosonic state.

\section{$2.1 \quad$ Spin 0}

The construction of the effective lagrangian for the spin-0 state is obtained by requiring that the parametrisation: i) allows one to recover the SM case easily; ii) has the possibility to include all possible interactions that are generated by gauge-invariant dimension-six operators above the EW scale; iii) includes $0^{-}$state couplings typical of SUSY or of generic two-Higgs-doublet models (2HDM); and iv) allows $C P$-mixing between $0^{+}$and $0^{-}$states (which we parametrise in terms of an angle $\alpha$ ). Let us comment on the second requirement, which is an important one. Our aim is that of using a formulation which is general enough to include all effects coming from dimension-six operators invariant under $\mathrm{SU}(2)_{L} \times \mathrm{U}(1)_{Y}$, i.e. above the EW scale. This results in a limited subset of all possible dimension-six operators [39, 40] that govern Higgs interactions. In addition, as a first step, we limit ourselves to include the operators that modify the three-point Higgs interactions. For the fermions, there is only one operator that modifies the Yukawa interaction, e.g. for the top quark, $\mathcal{L}_{Y}^{\operatorname{dim}=6}=\left(\phi^{\dagger} \phi\right) Q_{L} \tilde{\phi} t_{R}$, where $Q_{L}$ is the $\mathrm{SU}(2)_{L}$ doublet $\left(t_{L}, b_{L}\right)$. As far as the interactions to vector bosons are concerned, a larger number of dimension-six operators can be written down; the framework we adopt is general enough to account for them all, even though for practical reasons at this stage the implementation includes only those affecting all possible three-point interactions with exactly one Higgs field. We point out that, for a $C P$-even state, this parametrisation is in one-to-one correspondence with those of refs. [19, 38] (see e.g. eq. (3.46) of ref. [38]) not including the terms in $\mathcal{L}_{F_{1}}$ and $\mathcal{L}_{F_{2}}$ which modify four-point interactions, and equivalent to eq. (3) of ref. [32]. For a $C P$-odd state this is equivalent to eq. (A.98) of ref. [38].

Let us start with the interaction lagrangian relevant to fermions which, while being extremely simple, illustrates our philosophy well. Such a lagrangian is:

$$
\mathcal{L}_{0}^{f}=-\sum_{f=t, b, \tau} \bar{\psi}_{f}\left(c_{\alpha} \kappa_{H f f} g_{H f f}+i s_{\alpha} \kappa_{A f f} g_{A f f} \gamma_{5}\right) \psi_{f} X_{0}
$$

where we use the notation:

$$
c_{\alpha} \equiv \cos \alpha, \quad s_{\alpha} \equiv \sin \alpha,
$$

and denote by $g_{H f f}=m_{f} / v\left(g_{A f f}=m_{f} / v\right)$ the strength of the scalar (pseudoscalar) coupling in the SM (in a $2 \mathrm{HDM}$ with $\tan \beta=1$ ). We point out that the constants $\kappa_{i}$ can be taken real without any loss of generality, except $\kappa_{H \partial W}$ in eq. (2.4). For simplicity, we have assumed that only the third-generation of fermions couple to the scalar state; extensions 
to the other families and flavour-changing structures are trivial to implement, which can be directly done by users of FEynRules. As mentioned above, the interaction of eq. (2.2) can also parametrise the effects of a $\mathcal{L}_{Y}^{\operatorname{dim}=6}=\left(\phi^{\dagger} \phi\right) Q_{L} \tilde{\phi} t_{R}$ operator. Note also that all requirements listed above are satisfied at the price of a small redundancy in the number of parameters. The SM is obtained when $c_{\alpha}=1$ and $\kappa_{H f f}=1$. The pseudoscalar state of a type-II $C P$-conserving $2 \mathrm{HDM}$ or SUSY is obtained by setting $s_{\alpha}=1$ and $\kappa_{A f f}=\cot \beta$ or $\kappa_{A f f}=\tan \beta$ for up or down components of the SU(2) fermion doublet, respectively. The parametrisation of $C P$ mixing is entirely realised in terms of the angle $\alpha$, i.e. independently of the parameters $\kappa_{i}$, so that many interesting cases, such as again $C P$-violation in generic 2HDM, can be covered.

The effective lagrangian for the interaction of scalar and pseudoscalar states with vector bosons can be written as follows:

$$
\begin{aligned}
\mathcal{L}_{0}^{V}=\{ & c_{\alpha} \kappa_{\mathrm{SM}}\left[\frac{1}{2} g_{H Z Z} Z_{\mu} Z^{\mu}+g_{H W W} W_{\mu}^{+} W^{-\mu}\right] \\
& -\frac{1}{4}\left[c_{\alpha} \kappa_{H \gamma \gamma} g_{H \gamma \gamma} A_{\mu \nu} A^{\mu \nu}+s_{\alpha} \kappa_{A \gamma \gamma} g_{A \gamma \gamma} A_{\mu \nu} \widetilde{A}^{\mu \nu}\right] \\
& -\frac{1}{2}\left[c_{\alpha} \kappa_{H Z \gamma} g_{H Z \gamma} Z_{\mu \nu} A^{\mu \nu}+s_{\alpha} \kappa_{A Z \gamma} g_{A Z \gamma} Z_{\mu \nu} \widetilde{A}^{\mu \nu}\right] \\
& -\frac{1}{4}\left[c_{\alpha} \kappa_{H g g} g_{H g g} G_{\mu \nu}^{a} G^{a, \mu \nu}+s_{\alpha} \kappa_{A g g} g_{A g g} G_{\mu \nu}^{a} \widetilde{G}^{a, \mu \nu}\right] \\
& -\frac{1}{4} \frac{1}{\Lambda}\left[c_{\alpha} \kappa_{H Z Z} Z_{\mu \nu} Z^{\mu \nu}+s_{\alpha} \kappa_{A Z Z} Z_{\mu \nu} \widetilde{Z}^{\mu \nu}\right] \\
& -\frac{1}{2} \frac{1}{\Lambda}\left[c_{\alpha} \kappa_{H W W} W_{\mu \nu}^{+} W^{-\mu \nu}+s_{\alpha} \kappa_{A W W} W_{\mu \nu}^{+} \widetilde{W}^{-\mu \nu}\right] \\
& \left.-\frac{1}{\Lambda} c_{\alpha}\left[\kappa_{H \partial \gamma} Z_{\nu} \partial_{\mu} A^{\mu \nu}+\kappa_{H \partial Z} Z_{\nu} \partial_{\mu} Z^{\mu \nu}+\left(\kappa_{H \partial W} W_{\nu}^{+} \partial_{\mu} W^{-\mu \nu}+\text { h.c. }\right)\right]\right\} X_{0},
\end{aligned}
$$

where the (reduced) field strength tensors are defined as follows:

$$
\begin{aligned}
& V_{\mu \nu}=\partial_{\mu} V_{\nu}-\partial_{\nu} V_{\mu} \quad\left(V=A, Z, W^{ \pm}\right), \\
& G_{\mu \nu}^{a}=\partial_{\mu} G_{\nu}^{a}-\partial_{\nu} G_{\mu}^{a}+g_{s} f^{a b c} G_{\mu}^{b} G_{\nu}^{c},
\end{aligned}
$$

and the dual tensor is:

$$
\widetilde{V}_{\mu \nu}=\frac{1}{2} \epsilon_{\mu \nu \rho \sigma} V^{\rho \sigma}
$$

The parametrisation of the couplings to vectors follows the same principles as that of the couplings to fermions. In particular, the mixing angle $\alpha$ allows for a completely general description of $C P$-mixed states. We stress here that while in general in a given model $C P$ violation depends on the whole set of possible interactions among the physical states and cannot be established by looking only at a sub sector [46], in our parametrisation $\alpha \neq 0$ or $\alpha \neq$ $\pi / 2$ (and non-vanishing $\kappa_{H f f}, \kappa_{A f f}, \kappa_{H V V}, \kappa_{A V V}$ ) implies $C P$ violation. This can be easily understood by first noting that in eq. (2.2) $\alpha \neq 0$ or $\alpha \neq \pi / 2$ always leads to $C P$ violation and that the corresponding terms in eq. (2.4) are generated via a fermion loop by the $X_{0} f f$ interaction. The $C P$-odd analogues of the operators in the last line of eq. (2.4) do vanish. 


\begin{tabular}{|lll|}
\hline parameter & reference value & description \\
\hline$\Lambda[\mathrm{GeV}]$ & $10^{3}$ & cutoff scale \\
$c_{\alpha}(\equiv \cos \alpha)$ & 1 & mixing between $0^{+}$and $0^{-}$ \\
$\kappa_{i}$ & 0,1 & dimensionless coupling parameter \\
\hline
\end{tabular}

Table 1. Model parameters.

\begin{tabular}{|rccccc|}
\hline$g_{X y y^{\prime}} \times v$ & $f f$ & $Z Z / W W$ & $\gamma \gamma$ & $Z \gamma$ & $g g$ \\
\hline$H$ & $m_{f}$ & $2 m_{Z / W}^{2}$ & $47 \alpha_{\mathrm{EM}} / 18 \pi$ & $C\left(94 \cos ^{2} \theta_{W}-13\right) / 9 \pi$ & $-\alpha_{s} / 3 \pi$ \\
$A$ & $m_{f}$ & 0 & $-4 \alpha_{\mathrm{EM}} / 3 \pi$ & $-2 C\left(8 \cos ^{2} \theta_{W}-5\right) / 3 \pi$ & $-\alpha_{s} / 2 \pi$ \\
\hline
\end{tabular}

Table 2. Values in units of $v$ taken by the couplings $g_{X y y^{\prime}} \cdot C=\sqrt{\frac{\alpha_{\mathrm{EM}} G_{F} m_{Z}^{2}}{8 \sqrt{2} \pi}}$.

In our implementation, the parameters listed in table 1 can be directly set by the user. The dimensionful couplings $g_{X y y^{\prime}}$ shown in table 2 are set so as to reproduce a SM Higgs and a pseudoscalar one in a $2 \mathrm{HDM}$ with $\tan \beta=1$. Note that in this case we have chosen $v$ as a reference scale instead of $\Lambda$. The main reason is simply that such operators appear at one-loop in the SM and therefore their values are non-zero even in absence of new physics. More precisely, the forms of the $g_{X V V^{\prime}}$ couplings given in table 2 are the same as those which are loop-induced in the SM, when computed by retaining only the top-quark and the $W$ boson contributions to the loops, and in the limit where their masses tend to infinity. These settings are adopted essentially because of their extremely simple analytic expressions (which, in fact, turn out to be excellent approximations for all the true loop-induced form factors, except for $g_{H Z \gamma}$ one, which underestimates the correct value of the full loop computation by a factor slightly larger than two). It is obvious that any generic value of these couplings, and in particular those induced by mass or higher-order corrections and by new-physics deviations from the SM predictions, can be accounted for by setting $\kappa_{i} \neq 1 .^{2}$

\subsection{Spin 1}

We now discuss how to build the most general interactions of a spin-1 resonance with SM particles. One way to proceed would be that of assigning $\mathrm{SU}(2)_{L} \times \mathrm{U}(1)_{Y}$ quantum numbers to the new vector, of writing all possible operators up to dimension six with SM fields, and then of re-expressing them in terms of the physical states below the EW scale, following exactly the same procedure as was used for the scalars above. To be fully general, however, one should consider different $\mathrm{SU}(2)_{L} \times \mathrm{U}(1)_{Y}$ gauge representations and mixings with the SM gauge bosons. A simpler approach is that of just writing the most general interactions at the weak scale, and of considering only those with the lowest canonical dimension. For simplicity we follow the latter approach.

\footnotetext{
${ }^{2}$ Note, however, that for the sake of simplicity and to normalize our results to the SM, we use $g_{H g g}^{\mathrm{NLO}}=$ $-\frac{\alpha_{s}}{3 \pi}\left(1+\frac{11}{4} \frac{\alpha_{S}}{\pi}\right)$ in our simulations at NLO in QCD (while no finite renormalisation is needed for the pseudoscalar, $\left.g_{A g g}^{\mathrm{NLO}}=g_{H g g}^{\mathrm{LO}}\right)$.
} 
The interaction lagrangian for the spin-1 boson with fermions is written as follows:

$$
\mathcal{L}_{1}^{f}=\sum_{f=q, \ell} \bar{\psi}_{f} \gamma_{\mu}\left(\kappa_{f_{a}} a_{f}-\kappa_{f_{b}} b_{f} \gamma_{5}\right) \psi_{f} X_{1}^{\mu}
$$

The $a_{f}$ and $b_{f}$ are the SM vector and axial-vector couplings, i.e. for the quarks:

$$
\begin{aligned}
a_{u} & =\frac{g}{2 \cos \theta_{W}}\left(\frac{1}{2}-\frac{4}{3} \sin ^{2} \theta_{W}\right), & b_{u} & =\frac{g}{2 \cos \theta_{W}} \frac{1}{2}, \\
a_{d} & =\frac{g}{2 \cos \theta_{W}}\left(-\frac{1}{2}+\frac{2}{3} \sin ^{2} \theta_{W}\right), & b_{d} & =-\frac{g}{2 \cos \theta_{W}} \frac{1}{2},
\end{aligned}
$$

and similarly for the leptons. The most general $X_{1} W W$ interaction at the lowest dimension can be written as follows (see ref. [47]):

$$
\begin{aligned}
\mathcal{L}_{1}^{W}= & i \kappa_{W_{1}} g_{W W Z}\left(W_{\mu \nu}^{+} W^{-\mu}-W_{\mu \nu}^{-} W^{+\mu}\right) X_{1}^{\nu}+i \kappa_{W_{2}} g_{W W Z} W_{\mu}^{+} W_{\nu}^{-} X_{1}^{\mu \nu} \\
& -\kappa_{W_{3}} W_{\mu}^{+} W_{\nu}^{-}\left(\partial^{\mu} X_{1}^{\nu}+\partial^{\nu} X_{1}^{\mu}\right) \\
& +i \kappa_{W_{4}} W_{\mu}^{+} W_{\nu}^{-} \widetilde{X}_{1}^{\mu \nu}-\kappa_{W_{5}} \epsilon_{\mu \nu \rho \sigma}\left[W^{+\mu}\left(\partial^{\rho} W^{-\nu}\right)-\left(\partial^{\rho} W^{+\mu}\right) W^{-\nu}\right] X_{1}^{\sigma},
\end{aligned}
$$

where $g_{W W Z}=-e \cot \theta_{W}$. Note, once again, that our effective field theory description lives at energy scales where EW symmetry $\mathrm{SU}(2)_{L} \times \mathrm{U}(1)_{Y}$ is broken to $\mathrm{U}(1)_{E M}$. This approach does not require to specify the transformation properties of $X_{1}$ with respect to the EW symmetry. The parametrisation above could also be used for describing $X_{1} Z \gamma$ interactions which, however, have not been implemented. In the case of $Z Z$, Bose symmetry implies a reduction of the possible terms and the interaction lagrangian reduces to [47, 48]:

$$
\mathcal{L}_{1}^{Z}=-\kappa_{Z_{1}} Z_{\mu \nu} Z^{\mu} X_{1}^{\nu}-\kappa_{Z_{3}} X_{1}^{\mu}\left(\partial^{\nu} Z_{\mu}\right) Z_{\nu}-\kappa_{Z_{5}} \epsilon_{\mu \nu \rho \sigma} X_{1}^{\mu} Z^{\nu}\left(\partial^{\rho} Z^{\sigma}\right)
$$

The first term can be rewritten in terms of the second one plus a term that vanishes if $\partial_{\mu} X_{1}^{\mu}=0$, which we do not assume (for example in the SM $\partial_{\mu} Z^{\mu} \neq 0$ for non-vanishing fermion masses). No effective lagrangian $\mathcal{L}_{1}^{\gamma}$ is introduced. Due to the Landau-Yang theorem $[49,50]$ no transition can occur between an on-shell vector and two massless identical vectors. However, for completeness, we discuss the possibility of an off-shell spin1 state contributing to the $g g \rightarrow \gamma \gamma$ amplitude in appendix A. Parity conservation implies that for $X_{1}=1^{-}$

$$
\kappa_{f_{b}}=\kappa_{V_{4}}=\kappa_{V_{5}}=0
$$

while for $X_{1}=1^{+}$

$$
\kappa_{f_{a}}=\kappa_{V_{1}}=\kappa_{V_{2}}=\kappa_{V_{3}}=0 .
$$

Note that the conditions on $\kappa_{V_{2}}$ and $\kappa_{V_{4}}$ are trivial when $V=Z$ (see eq. (2.12)). 


\section{$2.3 \quad$ Spin 2}

The interaction lagrangian for the spin-2 boson proceeds via the energy-momentum (EM) tensor of the SM fields and starts at dimension five [51, 52]. For a colour, weak and electromagnetic singlet spin-2 resonance such an interaction is unique. For the fermions we have

$$
\mathcal{L}_{2}^{f}=-\frac{1}{\Lambda} \sum_{f=q, \ell} \kappa_{f} T_{\mu \nu}^{f} X_{2}^{\mu \nu}
$$

and analogously for the vector bosons

$$
\mathcal{L}_{2}^{V}=-\frac{1}{\Lambda} \sum_{V=Z, W, \gamma, g} \kappa_{V} T_{\mu \nu}^{V} X_{2}^{\mu \nu}
$$

The coupling parameters $\kappa_{f}$ and $\kappa_{V}$ are introduced $[43,53]$ in full analogy with what has been done in the spin- 0 and -1 cases. All of the E-M tensors $T_{\mu \nu}^{f, V}$ are given e.g. in refs. $[52,54]$. For the sake of later discussion, we explicitly present the E-M tensor for QED:

$$
\begin{aligned}
T_{\mu \nu}^{f}= & -g_{\mu \nu}\left[\bar{\psi}_{f}\left(i \gamma^{\rho} D_{\rho}-m_{f}\right) \psi_{f}-\frac{1}{2} \partial^{\rho}\left(\bar{\psi}_{f} i \gamma_{\rho} \psi_{f}\right)\right] \\
& +\left[\frac{1}{2} \bar{\psi}_{f} i \gamma_{\mu} D_{\nu} \psi_{f}-\frac{1}{4} \partial_{\mu}\left(\bar{\psi}_{f} i \gamma_{\nu} \psi_{f}\right)+(\mu \leftrightarrow \nu)\right], \\
T_{\mu \nu}^{\gamma}= & -g_{\mu \nu}\left[-\frac{1}{4} A^{\rho \sigma} A_{\rho \sigma}+\partial^{\rho} \partial^{\sigma} A_{\sigma} A_{\rho}+\frac{1}{2}\left(\partial^{\rho} A_{\rho}\right)^{2}\right] \\
& -A_{\mu}^{\rho} A_{\nu \rho}+\partial_{\mu} \partial^{\rho} A_{\rho} A_{\nu}+\partial_{\nu} \partial^{\rho} A_{\rho} A_{\mu}
\end{aligned}
$$

where $D_{\mu}=\partial_{\mu}-i e Q_{f} A_{\mu}$ and $A_{\mu \nu}=\partial_{\mu} A_{\nu}-\partial_{\nu} A_{\mu}$. For $X_{2}=2^{+}$in the minimal RS-like graviton scenario [55], i.e. the universal coupling strength to the matter and gauge fields, the parameters should be chosen as follows:

$$
\kappa_{f}=\kappa_{V} \quad \forall \quad f, V .
$$

\section{$3 \quad$ Validation and comparisons}

The implementation of the lagrangian $\mathcal{L}_{H C, J}$ in FeYnRules [42] allows the automated generation of the corresponding Feynman rules which can in turn be exported to the MADGrAPH 5 [56] framework via the UFO model file [44, 57]. This opens the possibility of automatically creating event-generator codes for any production and decay channel (including interferences between such two mechanisms) at the tree level, which can then be used standalone (i.e., at the parton level) or interfaced with parton-shower MCs (ME+PS). The same automated generation can be achieved to NLO accuracy (where the matching with showers is done according to the MC@NLO formalism [58]), with the present exception: for a user-defined lagrangian, as is the case here, one-loop corrections in some cases have to be provided externally — we shall give more details on this point in section 3.2. 


\begin{tabular}{|ccc|}
\hline JHU scenario & \multicolumn{2}{c|}{ HC parameter choice } \\
& $X$ production & $X$ decay \\
\hline $0_{m}^{+}$ & $\kappa_{H g g} \neq 0$ & $\kappa_{\mathrm{SM}} \neq 0\left(c_{\alpha}=1\right)$ \\
$0_{h}^{+}$ & $\kappa_{H g g} \neq 0$ & $\kappa_{H \gamma \gamma, H Z Z, H W W} \neq 0\left(c_{\alpha}=1\right)$ \\
$0^{-}$ & $\kappa_{A g g} \neq 0$ & $\kappa_{A \gamma \gamma, A Z Z, A W W} \neq 0\left(c_{\alpha}=0\right)$ \\
$1^{+}$ & $\kappa_{f_{a}, f_{b}} \neq 0$ & $\kappa_{Z_{5}, W_{5}} \neq 0$ \\
$1^{-}$ & $\kappa_{f_{a}, f_{b}} \neq 0$ & $\kappa_{Z_{3}, W_{3}} \neq 0$ \\
$2_{m}^{+}$ & $\kappa_{g} \neq 0$ & $\kappa_{\gamma, Z, W} \neq 0$ \\
\hline
\end{tabular}

Table 3. Parameter correspondence to the benchmark scenarios defined in table I of ref. [16]. In each scenario, the $\kappa_{i}$ couplings that are not explicitly mentioned are understood to be equal to zero.

\subsection{Leading-order parton-level results}

We start by considering the most elementary type of predictions our approach is capable of giving, namely those at the Born level without parton showers (i.e., processes that do not feature any final-state particle either different from $X\left(J^{P}\right)$, or not resulting from the $X\left(J^{P}\right)$ decay). Thus, this only involves the FEynRules - UFO - MAdGraph 5 chain, which by now has been applied to hundreds of processes and is therefore extremely well tested. Still, it is appropriate to check the results of the Higgs Characterisation model, in particular in view of other implementations available in the literature that aim at describing the same leading-order physics, and specifically that of JHU [16].

In table 3 we give the choices of parameters to be made in order to obtain the benchmarks defined in ref. [16]. For all scenarios listed in that table we have found complete agreement in the mass and angular distributions of the $X\left(J^{P}\right)$ decay products: $Z Z, W W$, and $\gamma \gamma$. For further studies to be made here, we employ the process $p p \rightarrow X\left(\rightarrow Z Z^{*}\right) \rightarrow 4 \ell$ to be definite, and we do not impose any final-state kinematical cuts.

We note that our $C P$-even spin-0 parametrisation also includes the so-called "derivative operators" (last line of eq. (2.4)), that are absent in the parametrisation of ref. [16], and that give non-trivial contributions to $X_{0} \rightarrow V V$ decays. In fact, by using the equations of motion, it can be easily seen that these operators can be related to contact $X_{0} V f f$ operators of the kind recently discussed in refs. [59,60]. A representative set of distributions for key spin-correlation observables is shown in figure 1 . One notices that the higher-dimensional operators corresponding to $\kappa_{H Z Z}\left(C P\right.$-even) and $\kappa_{A Z Z}(C P$-odd) have dramatic effects on angular distributions, such as those of $\cos \theta_{1}, \Delta \phi$, while the derivative operators corresponding to $\kappa_{H \partial Z}$ only (mildly) affect the lepton invariant mass distributions $m_{1}$ and $m_{2}$.

For spin 1, we remark that the $X_{1} V V$ interactions defined in ref. [16] have one-toone correspondence with the $\kappa_{V_{3}}$ and $\kappa_{V_{5}}$ terms for both the $X_{1} W W$ and $X_{1} Z Z$ cases. However, eqs. (2.11) and (2.12) show that in general the $X_{1} V V$ vertices can have a richer structure. Sample distributions are shown in figure 2 for $X_{1} \rightarrow Z Z$, where the difference between $1^{+}$and $1^{-}$are manifest. 

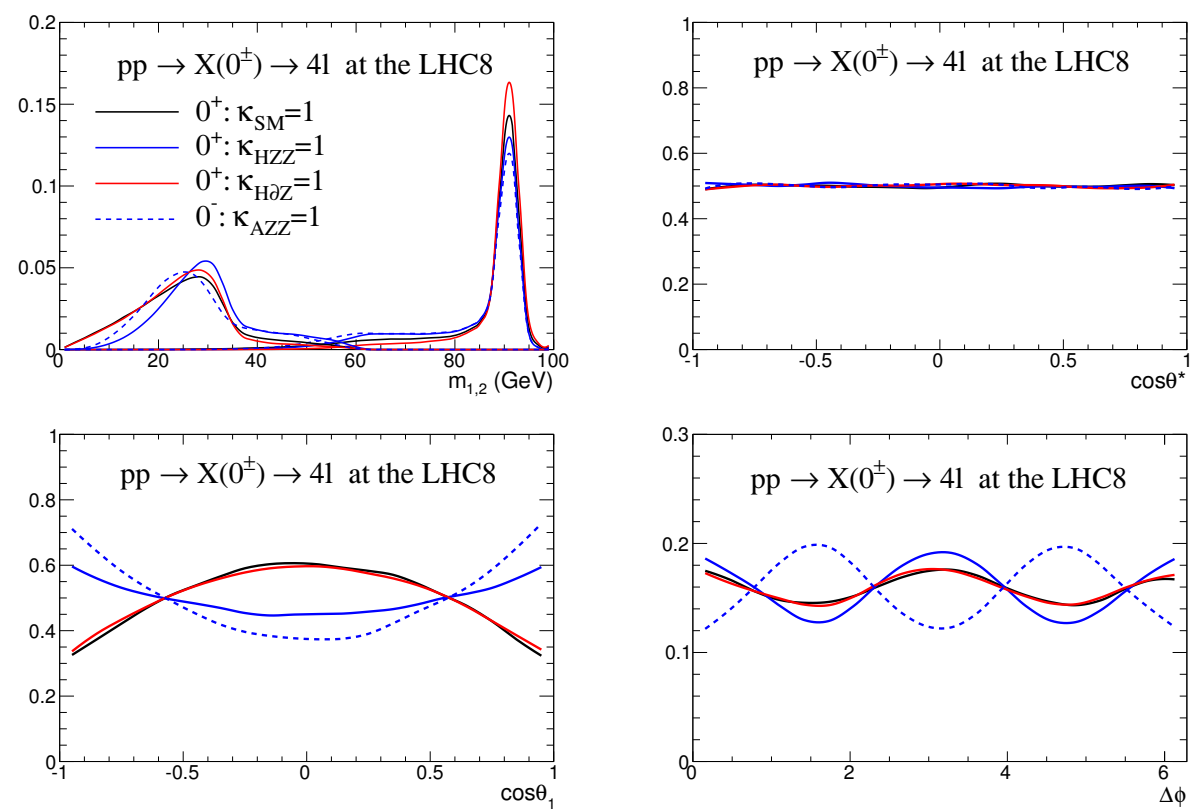

Figure 1. Normalised distributions in $p p \rightarrow X_{0} \rightarrow \mu^{+} \mu^{-} e^{+} e^{-}$for different choices of $X_{0} Z Z$ couplings: the invariant masses of the two lepton pairs $m_{1}, m_{2}\left(\right.$ with $\left.m_{1}>m_{2}\right), \cos \theta^{*}, \cos \theta_{1}$, and $\Delta \phi$, as defined in ref. [16]. Event simulation performed at the leading order, parton level only (no shower/hadronisation).
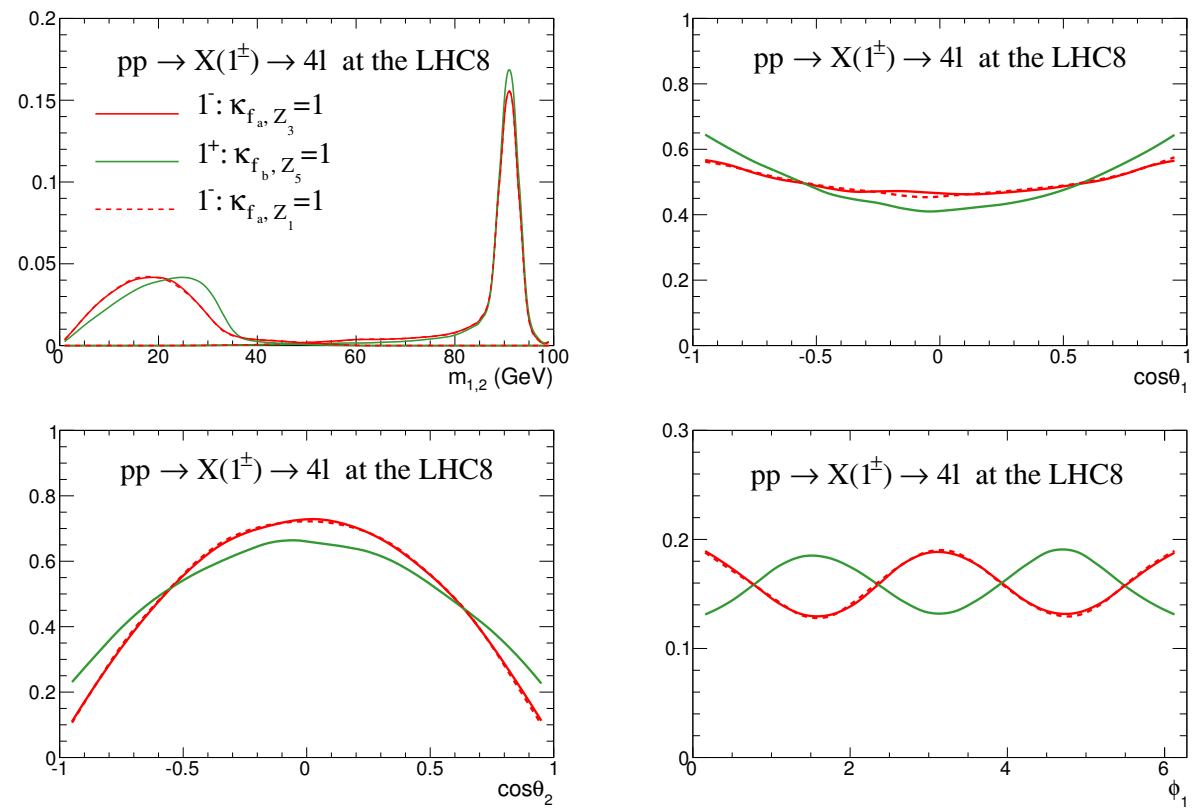

Figure 2. Normalised distributions in $p p \rightarrow X_{1} \rightarrow \mu^{+} \mu^{-} e^{+} e^{-}$for different choices of $X_{1} Z Z$ couplings: the invariant masses of the two lepton pairs $m_{1}, m_{2}\left(\right.$ with $\left.m_{1}>m_{2}\right), \cos \theta_{1}, \cos \theta_{2}$, and $\phi_{1}$, as defined in ref. [16]. Event simulation performed at the leading order, parton level only (no shower/hadronisation). 

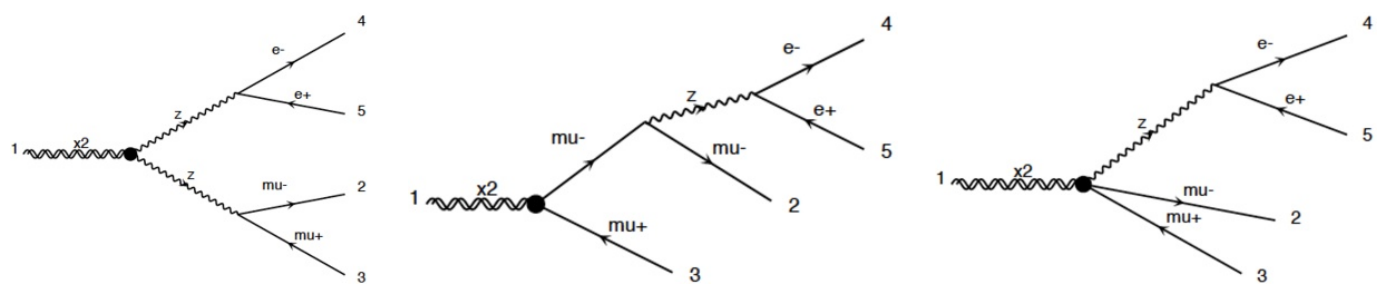

Figure 3. Representative diagrams for the decay of $X_{2} \rightarrow 4 \ell$.
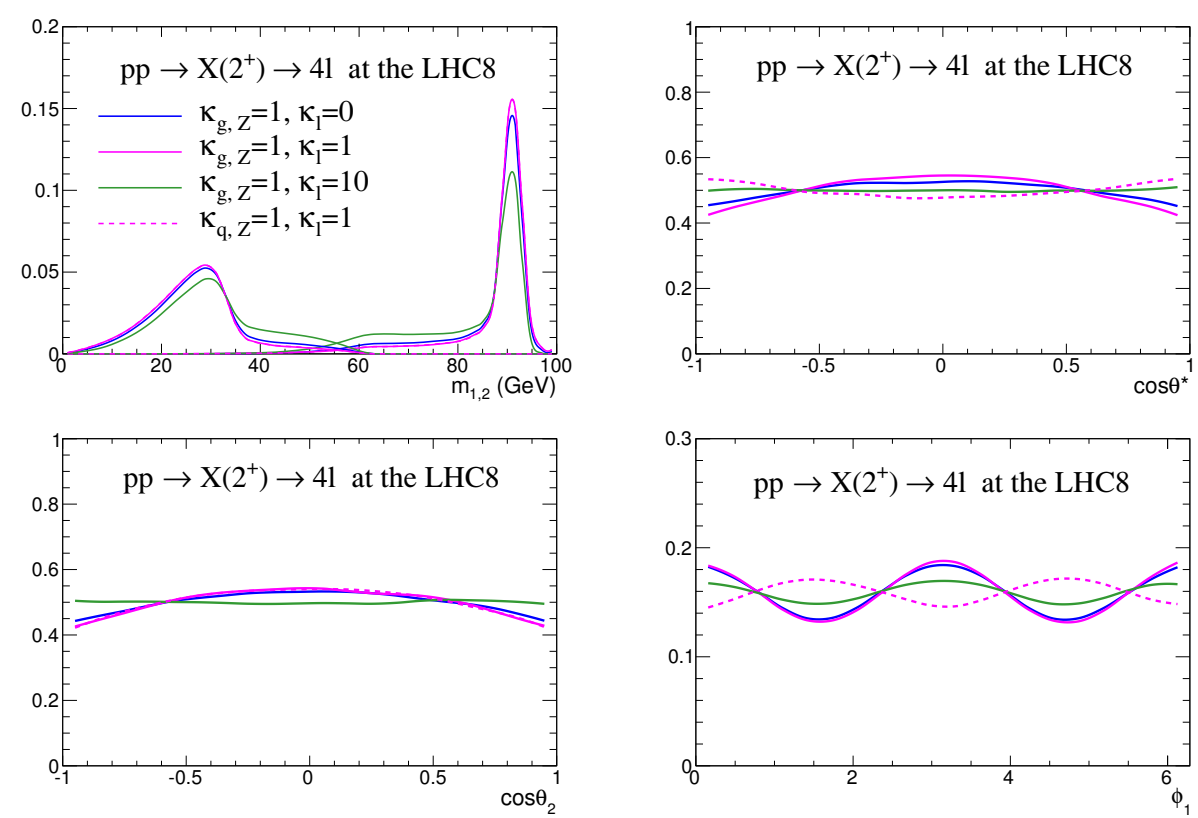

Figure 4. Normalised distributions in $p p \rightarrow X_{2} \rightarrow \mu^{+} \mu^{-} e^{+} e^{-}$for different $\kappa_{\ell}$ values: the invariant masses of the two lepton pairs $m_{1}, m_{2}$ (with $m_{1}>m_{2}$ ), $\cos \theta^{*}, \cos \theta_{2}$, and $\phi_{1}$, as defined in ref. [16]. Event simulation performed at the leading order, parton level only (no shower/hadronisation).

For spin 2, our minimal approach consists of sticking to the minimal five-dimensional interaction and of imposing the invariance of $\mathcal{L}_{2}$ under the gauge symmetries of the SM. As a result, in the case of universal couplings to SM particles $X_{2}$ is equivalent to a minimal RS-graviton. As it will be discussed in the following, a spin-2 state with non-universal couplings to SM particles might have a very different behaviour with respect to that of an RS-graviton, especially at high energies. In order to further this point, in figure 3 we show some of the diagrams involved in the decay $X_{2} \rightarrow 4 \ell$. Were the resonance above twice the $Z$ mass, one could certainly only consider the first diagram, which would be by far the dominant one. For a mass around $125 \mathrm{GeV}$, however, one of the $Z$-bosons is not on-shell and diagrams such as the second and third one become relevant and need to be included. In figure 4 the dependence on the coupling $\kappa_{\ell}$ that appears in eq. (2.15) of key distributions, i.e. the invariant mass of the lepton pairs $m_{1}$ and $m_{2}$ (with $m_{1}>m_{2}$ ), $\cos \theta^{*}$, $\cos \theta_{2}$, and $\phi_{1}$ distributions (see ref. [16] for their definition), is shown. The magenta lines are the case for $\kappa_{Z}=\kappa_{\ell} \neq 0$ with the $g g$ initial state $\left(\kappa_{g} \neq 0\right.$, solid $)$ and the $q \bar{q}\left(\kappa_{q} \neq 0\right.$, 
dotted). The most striking differences are seen for the case where the spin-2 coupling to the fermions is enhanced by a factor of 10 (green line).

\subsection{Higher orders in QCD}

The LO predictions previously discussed can be systematically improved by including the effects due to the emission of QCD partons; this can be done by considering both tree-level and full-NLO matrix elements, and their matching with parton showers.

The ME+PS simulations are based on tree-level matrix elements for production and decays, and allow one to retain all spin correlations. Extra jet radiation can be realistically taken into account by merging matrix elements with different parton multiplicities with parton shower programs, such as HERWIG [61, 62] or Pythia [63, 64]. The MadGraph 5 platform features an interface with PYTHIA6.4 [63] that makes use of the MLM- $k_{T}[65,66]$, and of the shower- $k_{T}$ [67] merging prescriptions. The two matching schemes have been tested in several cases and shown to give equivalent results (see e.g. refs. [67, 68]). Studies presented in this work are performed using the MLM- $k_{T}$ matching scheme.

AMC@NLO is an event generator that implements the matching of any NLO QCD computation with parton showers according to the MC@NLO formalism [58], and which is embedded in the MADGRAPH 5 framework. It is based on two main building blocks, each devoted to the generation and evaluation of a specific contribution to an NLO-matched computation. MADFKS [69] deals with the Born and real-emission amplitudes, and in particular it performs, according to the FKS prescription [70, 71], the subtraction of the infrared singularities that appear in the latter matrix elements; moreover, it is also responsible for the generation of the so-called Monte Carlo subtraction terms, namely the contributions that prevent any double-counting in the MC@NLO cross sections. MADLOOP [72] computes the one-loop amplitudes, using the OPP [73] integrand-reduction method and its implementation in CuTTools [74]. These procedures are fully automated (hence, they do not require any coding by the user, with the relevant computer codes being generated on-the-fly), provided that a basic knowledge is available about the underlying theory and the interactions of its particles with QCD partons. For MADFKS this amounts to the ordinary Feynman rules; for MADLoop, to Feynman rules, UV counterterms, and special tree-level rules necessary to, and defined by, the OPP method, which are called $R_{2}$. While Feynman rules are automatically computed given the lagrangian (via FEYNRULES), this is not yet possible for the UV counterterms and $R_{2}$ rules. ${ }^{3}$ The solution adopted thus far is that of coding by hand these pieces of information, for all cases where the relevant analytical computations had already been carried out, namely QCD and EW corrections in the SM [75-78] and for QCD corrections in SUSY models [79].

The upshot of this is the following: for the Higgs characterisation model, all the ingredients entering the MC@NLO cross sections can be computed automatically, except for (some of) the one-loop matrix elements. In order to amend the latter issue, one can choose either of the following two strategies: that of computing analytically the relevant UV counterterms and $R_{2}$ rules, and of implementing them in the appropriate UFO module; or

\footnotetext{
${ }^{3}$ However, a preliminary version of FEYNRULES exists which does exactly this.
} 
that of computing directly the relevant one-loop matrix elements. While the former strategy has a broader scope, the (considerable) effort it entails is not justified in view of the progress with FeynRules mentioned above. Hence, the latter strategy is quicker to pursue, and less error-prone in the short term. This is because it can rely on results readily available in the literature. For $p p \rightarrow X_{0}+$ anything, the one-loop matrix elements for both the $0^{+}$and $0^{-}$ states have been known since a long time [80,81]. Results for the production of a $C P$-mixed state can also be easily obtained, even though this scenario is not yet implemented. The case of $p p \rightarrow X_{1}+$ anything is exactly the same as Drell-Yan. For the inclusive production of a spin-2 boson, the analytic results for the virtual amplitudes of refs. [82-89] have been extended to allow for non-universal couplings to quarks and gluons. Their implementations in AMC@NLO includes spin correlated decays to $\gamma \gamma, W^{+} W^{-}$, and $Z Z$ to four leptons. All the three classes of matrix elements mentioned here have been implemented by hand in AMC@NLO, and used for the simulations presented in this paper. We point out that, in the case of vector boson fusion (VBF) and of vector-boson associated production, all NLO computations can be done automatically and in full generality, ${ }^{4}$ with the exception of the spin-2 case, which is feasible provided that one assumes vanishing couplings with QCD particles. Studies, such as those presented in refs. [43, 90, 91] could therefore be performed at NLO accuracy. Finally, we mention that Level-2 studies in $t \bar{t}$ associated production can be performed for spin-0 [92] and spin-1 [69] in a fully automatic way.

\subsubsection{Inclusive production $p p \rightarrow X\left(J^{P}\right):$ ME+PS vs. aMC@NLO}

As is well known, the ME+PS and MC@NLO approaches often give complementary benefits. In those phase-space regions where both of them are sensible, it is interesting to compare their predictions, as a way towards their validation through a mutual consistency check. To this end, in this section we present the results of a comparison between ME+PS and AMC@NLO for the case of inclusive $X\left(J^{P}\right)$ production, with $J^{P}=0^{+}, 0^{-}, 1^{+}, 1^{-}$, and $2^{+}$. In the following analyses, we generate events at the LHC with a center-of-mass energy $\sqrt{s}=8 \mathrm{TeV}$ and assume the mass of the new boson to be $m_{X}=125 \mathrm{GeV}$. ME+PS merged samples consist of events at the matrix element level for $p p \rightarrow X+0,1,2$ partons obtained with MAdGraph 5, with parameters $Q_{\mathrm{min}}^{\mathrm{ME}}=40 \mathrm{GeV}$ and $Q_{\min }^{\text {jet }}=25 \mathrm{GeV}$, and with CTEQ6L1 [93] PDFs. These samples are then showered with Pyтнia 6.4 ( $p_{T}$-ordered) by using the MLM- $k_{T}$ scheme for merging. The AMC@NLO samples are obtained by setting the renormalisation and factorisation scales equal to $m_{X}$ and by employing MSTW2008 NLO PDFs [94] for the short-distance calculation. Both LO and NLO samples are showered with the default parameter settings in PYTHIA (including the PDFs, CTEQ5L [95]), in order to be mostly sensitive to differences arising at the level of matrix elements. After shower and hadronisation, final state particles are clustered into jets using the anti- $k_{T}$ algorithm [96] (as implemented in FASTJET [97]) with radius parameter $\Delta R=0.4$. Jets are required to have a transverse momentum $p_{T}^{j}>25 \mathrm{GeV}$.

\footnotetext{
${ }^{4}$ Because the corresponding one-loop amplitudes are trivial, and do not necessitate any UV or $R_{2}$ information from the Higgs-characterisation lagrangian — in the case of VBF, this assumes that the pentagon contributions are discarded, as is customary in the SM.
} 
We start by presenting distributions for the transverse momentum and pseudorapidity of the new boson, as well as for the exclusive jet multiplicity (see figure 5). Both the $p_{T}^{X}$ and $\eta^{X}$ distributions roughly fall into two classes, determined by the dominant production mechanism at the LO ( $g g$ or $q \bar{q})$ - gluon fusion ( $q \bar{q}$ annihilation) accounts for $100 \%(0 \%)$, $0 \%(100 \%)$, and $96 \%(4 \%)$ in the case of the production of a spin-0, spin- 1 , and spin-2 with universal couplings (i.e., an RS graviton) state, respectively. Processes dominated by $g g$ fusion display a harder $p_{T}$ spectrum and are more central than the $q \bar{q}$-dominated ones. The rapidity difference is easily understood by the fact that at a $p p$ collider the $q$ are valence quarks while the $\bar{q}$ are from the sea and therefore configurations with asymmetric Bjorken $x$ 's for the two partons are more frequent. Another important observation is that the inclusive distributions for a spin- 0 and spin- 2 are indeed very similar, i.e. the spin has no real relevance for these observables. In the lower insets the bin-by-bin ME+PS over AMC@NLO ratios are shown. These ratios, computed by first normalising the corresponding distributions to unity, only convey shape information. It is manifest that the two methods give very similar predictions, both in $p_{T}^{X}$ and $\eta^{X}$. Differences in the $p_{T}^{X}$ spectra start to be significant above $m_{X}$, and in particular the merged samples produce a bit harder spectra for very large $p_{T}^{X}$ 's. This is obviously the effect of the larger amount of hard radiation in the ME+PS samples, which is in turn due to the presence there of the $p p \rightarrow X+2$ partons matrix elements, which are not included in the AMC@NLO predictions. This is also the reason why the exclusive jet multiplicities, shown in the plot at the bottom of figure 5 , are larger when $n>1$ jets in the case of the merged samples than when computed with AMC@NLO.

In the context of an automated approach, taking into account of the spin correlations relevant to the $X\left(J^{P}\right)$ decay products simply amounts to generating the process with those decay products as final states (the presence of $X\left(J^{P}\right)$ as an intermediate particle can also be imposed). From the general discussion given above, it should be clear that this is always feasible in the case of the ME+PS approach, while AMC@NLO may be limited by the availability of the one-loop matrix elements. However, spin 0 is obviously a trivial case (a spinless particle does not induce spin correlations). On the other hand, in the spin-1 and spin-2 cases the spin-correlated virtuals have been calculated; this is rather easy to do, since their expressions factorise the underlying Born matrix elements. We have then compared many key distributions as predicted by ME+PS and AMC@NLO, and have always found a satisfactory agreement. For the sake of illustration we show in figures 6,7 and 8 the results for a few selected final states of special interest, i.e. $X \rightarrow \gamma \gamma, X\left(\rightarrow Z Z^{*}\right) \rightarrow 4 \ell$, and $X\left(\rightarrow W W^{*}\right) \rightarrow 2 \ell 2 \nu$. We have imposed minimal acceptance cuts on the photons and charged leptons, namely:

$$
p_{T}^{\gamma, \ell}>5 \mathrm{GeV}, \quad\left|\eta^{\gamma, \ell}\right|<2.5
$$

The $\gamma \gamma$-case plots (figure 6) suggest that a good discriminating power between the spin-0 and spin-2 cases can be obtained from the $p_{T}$ distributions. Figure 7 illustrates the different shapes in $p_{T}$ and invariant mass of the two reconstructed $Z^{\prime} \mathrm{s}\left(Z_{1}\right.$ being the one with the largest invariant mass) for the different spin and parity hypotheses. As already noted in the literature $[16,98]$ the lowest pair invariant mass is particularly sensitive to both spin 

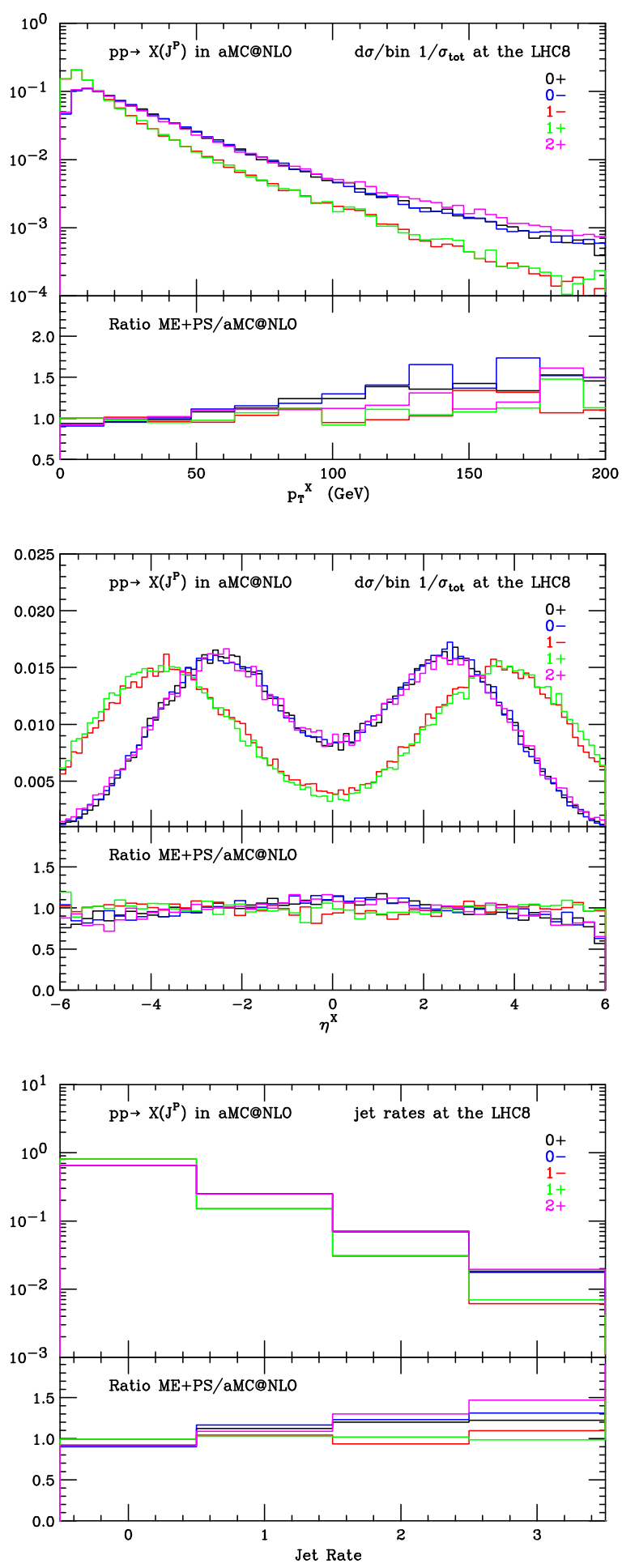

Figure 5. The transverse momentum $p_{T}^{X}$, pseudorapidity $\eta^{X}$, and jet rates of the new boson $X\left(J^{P}\right)=0^{+}, 0^{-}, 1^{+}, 1^{-}, 2^{+}$as obtained from AMC@NLO. The lower inset shows the bin-by-bin ratio of the same distribution obtained via ME+PS merging and that of AMC@NLO. 

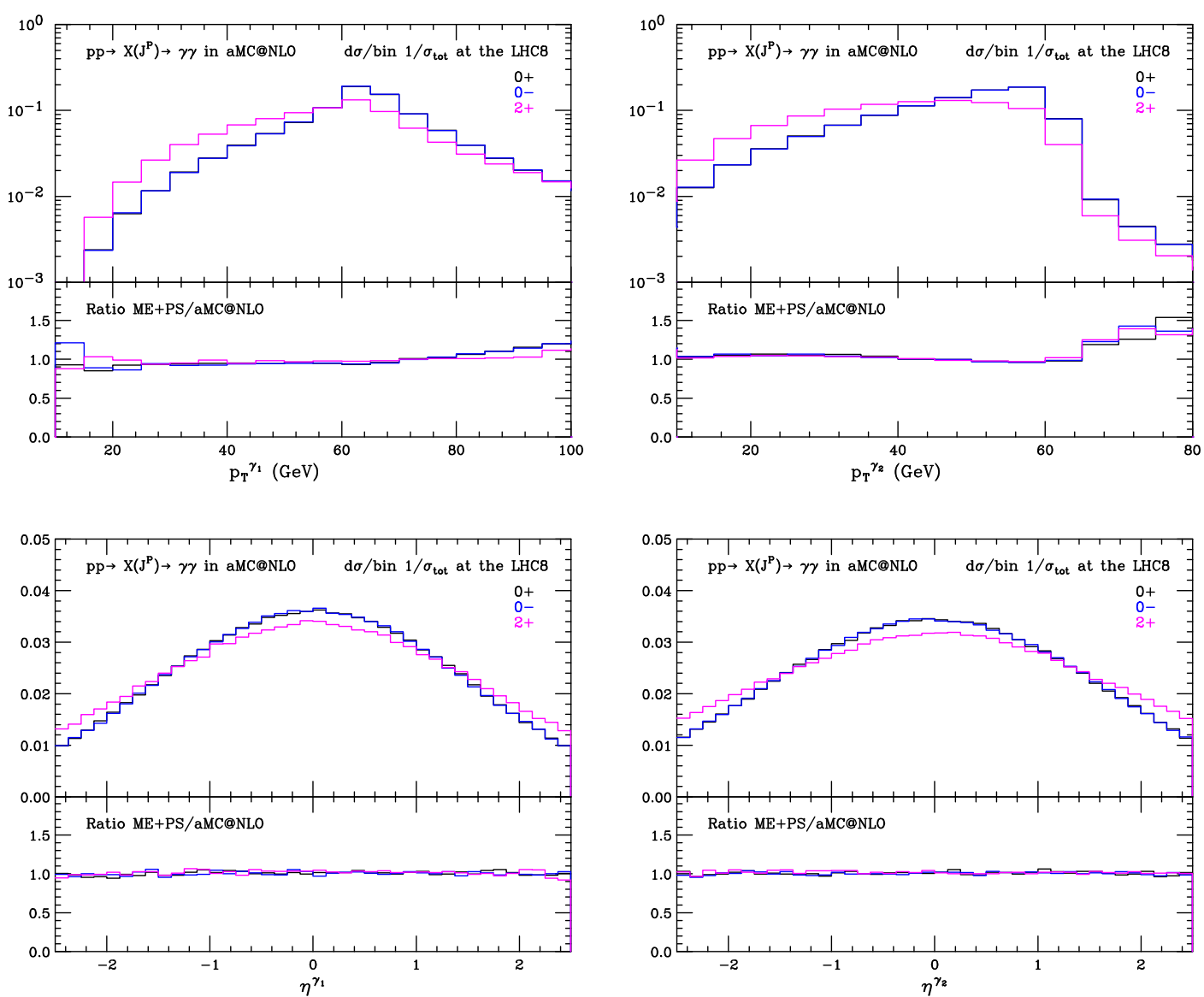

Figure 6. Distributions in $X \rightarrow \gamma \gamma$ : (a) and (b) the transverse momentum of the leading and subleading photon, $p_{T}^{\gamma_{1}}$ and $p_{T}^{\gamma_{2}},(\mathrm{c})$ and (d) the rapidity of the leading and subleading photon, $\eta^{\gamma_{1}}$ and $\eta^{\gamma_{2}}$.

and parity assignments. Finally, the transverse momentum of one of the charged leptons and the invariant mass distributions of the two charged leptons in the $W W^{*}$ channel are shown in figure 8 . The lepton $p_{T}$ distribution is sensitive to initial state radiation and it is harder at large $p_{T}$ 's in the case of the spin- 0 and spin- 2 hypotheses, reflecting the different $p_{T}^{X}$ shapes of such cases w.r.t. that resulting from $X\left(1^{ \pm}\right)$production.

The overall agreement between the predictions of ME+PS and AMC@NLO is rather good for all those observables that are not sensitive to hard radiation of at least two extra partons with respect to the Born kinematics. Other visible differences are mostly related to the harder $p_{T}^{X}$ spectra of the ME+PS samples (as documented in the upper plot of figure 5), which result in the enhancement in $p_{T}^{\gamma_{2}}$ (and to less extent also in $p_{T}^{\gamma_{1}}$ ) above the kinematic threshold $m_{X} / 2$ (see the first two plots of figure 6 ), and in $p_{T}^{Z_{1,2}}$ as well (see the first two plots of figure 7 ). 

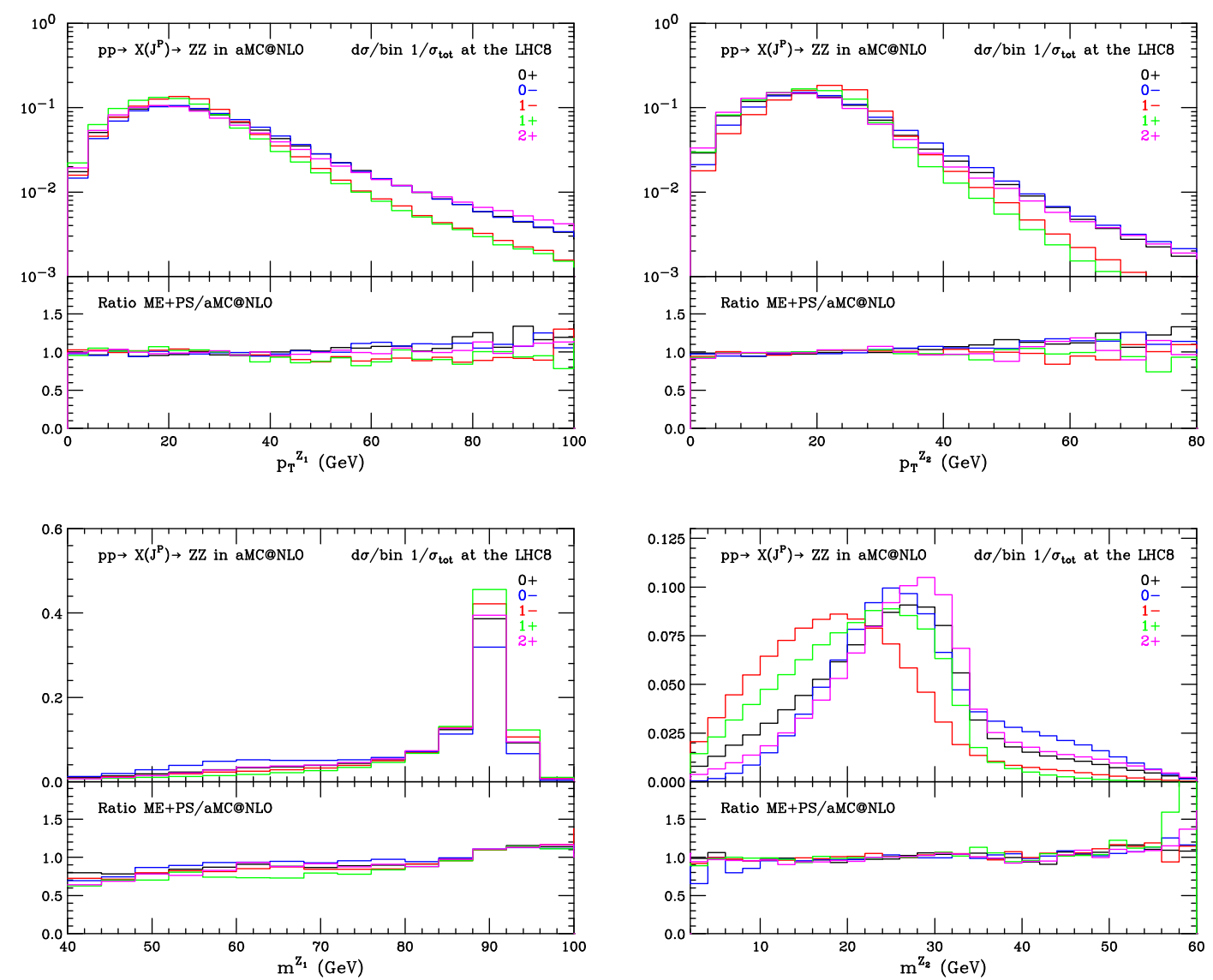

Figure 7. Distributions of the $Z$ bosons in $X\left(\rightarrow Z Z^{*}\right) \rightarrow \mu^{+} \mu^{-} e^{+} e^{-}:$(a) and (b) the transverse momentum of the $Z$ boson with the highest and lowest reconstructed mass, $p_{T}^{Z_{1}}$ and $p_{T}^{Z_{2}}$, (c) and (d) the invariant mass of the two leptons $m_{\ell \ell}$ corresponding to $Z_{1}$ and $Z_{2}$.

\section{Applications}

\subsection{Unitarity-violating behaviour of models with a spin-2 state}

In this section we discuss the behaviour of a spin-2 state with non-universal couplings to SM particles, i.e. with different $\kappa_{i}$ in the $\mathcal{L}_{2}$ lagrangian (in other words, eq. (2.19) does not hold here). The interest for this case comes from the fact that a model that features an RS-graviton with a mass of $125 \mathrm{GeV}$ and universal couplings has been already excluded at the Tevatron [99-101]. In addition, the current measured branching ratios and cross sections impose a very clear pattern in the values of couplings [53, 102, 103]. It is therefore important to investigate the effects of setting the couplings to non-equal values (non-universal scenario), in particular for what concerns the stability of the effective field theory with respect to higher order corrections. The first important point to realize is that couplings can be changed without breaking any of the gauge symmetries of the SM, as one can explicitly check by inspecting the E-M tensor for QED (eqs. (2.17) and (2.18)), which is manifestly invariant under a gauge transformation of the fermion and $A^{\mu}$ fields. 

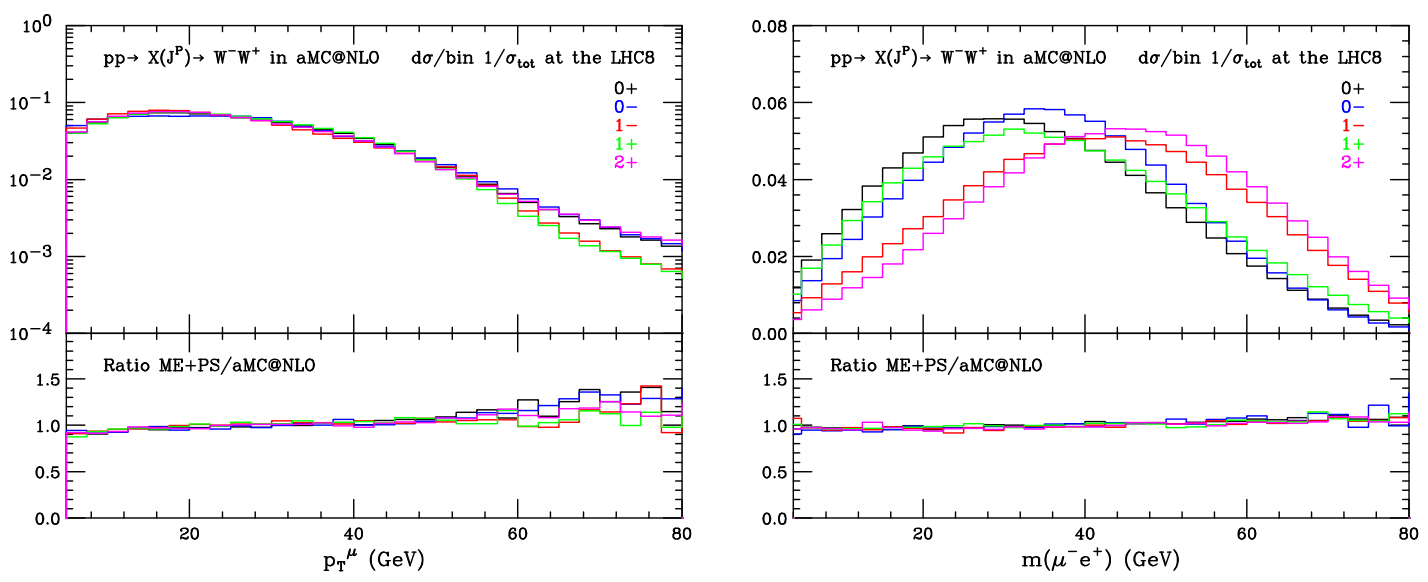

Figure 8. Distributions of the leptons in $X\left(\rightarrow W W^{*}\right) \rightarrow \mu^{-} \bar{\nu}_{\mu} e^{+} \nu_{e}$ : (a) the transverse momentum of the muon, $p_{T}^{\mu}$, (b) the invariant mass of the two leptons $m\left(\mu^{-} e^{+}\right)$.

In so doing, however, the spin-2 current is not conserved anymore, which can also be easily checked. In the case at hand, i.e. of a theory with a massive spin-2 state, this poses no problem of principle. It has, on the other hand, important effects in the behaviour of the scattering amplitudes at high energy, as we shall now explicitly show.

As was already mentioned, in the case of an RS graviton (universal couplings) the LO cross section is dominated by the $g g$ production channel $(96 \%$ vs. $4 \%$ due to the $q \bar{q}$ contribution). It is tempting to explore the case where this hierarchy is inverted, by tuning the parameters $\kappa_{q}$ and $\kappa_{g}$ that enter in the couplings of the graviton with the E-M tensor of quarks and gluons:

$$
\mathcal{L}=-\frac{1}{\Lambda} \kappa_{q} T_{\mu \nu}^{q} X_{2}^{\mu \nu}-\frac{1}{\Lambda} \kappa_{g} T_{\mu \nu}^{g} X_{2}^{\mu \nu}
$$

Note that while $T_{\mu \nu}^{g}$ contains only gauge fields, the first term $T_{\mu \nu}^{q}$ involves a coupling of fermionic fields with the gauge field through the covariant derivative. $T_{\mu \nu}^{q}$ and $T_{\mu \nu}^{g}$ are separately $\mathrm{SU}(3)_{C}$ gauge invariant.

At the NLO, Born and virtual $2 \rightarrow 1$ and real $2 \rightarrow 2$ contributions need to be taken into account. As it has been already noted in several papers (see e.g. [83, 89, 104, 105]), when $\kappa_{q}=\kappa_{g}$ all the UV divergences present in the intermediate stages of an NLO calculation cancel with the standard UV counterterms, and no additional overall renormalisation is required. This property is a consequence of the conservation of the E-M tensor. For non-universal couplings this is not the case anymore: UV divergences appear and therefore loop amplitudes need to be renormalised. The details of this procedure are given in appendix B. As far as the real emission contributions are concerned, they are associated with the processes $g g \rightarrow X_{2} g$ and $q \bar{q} \rightarrow X_{2} g$ (plus their crossings). The $g g \rightarrow X_{2} g$ amplitude depends only on $\kappa_{g}$, and therefore there is no impact on this amplitude from the non-conservation of the spin-2 current. On the other hand, a unitarity-violating behaviour stems from the $q \bar{q} \rightarrow X_{2} g$ amplitude (and its crossings). In fact, this amplitude contains three diagrams proportional to $\kappa_{q}$ and one proportional to $\kappa_{g}$. A calculation of such an 
amplitude gives:

$$
\begin{aligned}
|\mathcal{M}|^{2}=\frac{N}{\Lambda^{2} s t u m^{4}}\{ & 3 \kappa_{g}^{2} m^{4}\left[2 m^{4}-2 m^{2}(t+u)+t^{2}+u^{2}\right]\left[m^{4}-m^{2}(t+u)+4 t u\right] \\
& +\left(\kappa_{q}-\kappa_{g}\right) 6 \kappa_{g} m^{4} s\left[m^{6}+m^{2} s(s+2 u)-2 s u(s+u)\right] \\
& +\left(\kappa_{q}-\kappa_{g}\right)^{2} s\left[6 m^{10}-6 m^{8}(t+u)+3 m^{6}\left(t^{2}+u^{2}\right)-12 m^{4} t u(t+u)\right. \\
& \left.\left.+2 m^{2} t u\left(t^{2}+12 t u+u^{2}\right)-2 t u\left(t^{3}+t^{2} u+t u^{2}+u^{3}\right)\right]\right\},
\end{aligned}
$$

where $N$ is a dimensionless function of the couplings, and $m$ is the mass of the $X_{2}$ state. Firstly, we note that for $\kappa_{q}=\kappa_{g}$ the expression above reduces to the well-known result for graviton production in extra-dimension scenarios $[51,52,106]$. Secondly, we stress that the soft and collinear limits of the amplitude in eq. (4.2) are the same as those of the universal-coupling case, $\kappa_{q}=\kappa_{g}$; in other words, the terms which arise when $\kappa_{q} \neq \kappa_{g}$ do not modify the IR behaviour w.r.t. the RS case, and thus the resulting divergences factorise over the corresponding $g g \rightarrow X_{2}$ or $q \bar{q} \rightarrow X_{2}$ amplitudes.

For $\kappa_{q}=\kappa_{g}$ the amplitude in eq. (4.2) grows with energy as $s / \Lambda^{2}$, i.e. with the scaling expected from a dimension-five interaction. On the other hand, the term proportional to $\left(\kappa_{q}-\kappa_{g}\right)^{2}$ grows as fast as $s^{3} / m^{4} \Lambda^{2}$. This term can be traced back to the longitudinal parts of the graviton polarisation tensor that decouple only when the graviton current is conserved, i.e. when $\kappa_{q}=\kappa_{g}$. It is easy to verify that with the couplings needed to reproduce the Higgs signal at the LHC energy the amplitude does not yet violate the unitarity bound, even though the $p_{T}^{X}$ spectrum is significantly affected. Such a growth is not present in the $2 \rightarrow 1$ amplitudes simply because at the leading order the two contributions $q \bar{q} \rightarrow X_{2}$ and $g g \rightarrow X_{2}$ are completely independent. In order to study these effects in a consistent way, we have extended the NLO calculation of ref. [89] to the non-universal case and have implemented it in AMC@NLO. As a striking example of the non-universality effects on the spin-2 production, we display in figure 9 the $p_{T}^{X}$ distributions of the spin- 2 state for various choices of the quark/gluon couplings. The rather flat tails in several of the distributions are an evident sign of the increased unitarity-violating behaviour of the scenarios with $\kappa_{q} \neq \kappa_{g}$. We note that the cases where one assumes that the spin-2 state is being produced either in the $g g$ - or in the $q \bar{q}$-initiated process give very different results w.r.t. those of the RS graviton scenario. As a further confirmation that the unitarity-violating behaviour is induced by short-distance cross sections with at least one final-state QCD parton, we have verified that the spectra obtained with $\mathrm{ME}+\mathrm{PS}$ display the same behaviour as those of AMC@NLO shown in figure 9, and in particular that yet higher parton multiplicities do not alter significantly the unitarity-violating behaviour of the $2 \rightarrow 2$ amplitudes.

\subsection{Higher order QCD effects on spin observables for a spin-2 state}

A generally interesting question is that of whether higher-order (QCD) corrections have a sizable impact on observables constructed to be particularly sensitive to spin-correlation effects. The expectation that they do not, owing to the fact that kinematics effects such as the recoil of the primary system against QCD radiation largely factor out in spin-correlation observables, may simply be too naive. One must in fact account for the possibility that matrix elements with larger (than Born) final-state multiplicities give rise to new helicity configurations that may significantly affect spin correlations. 


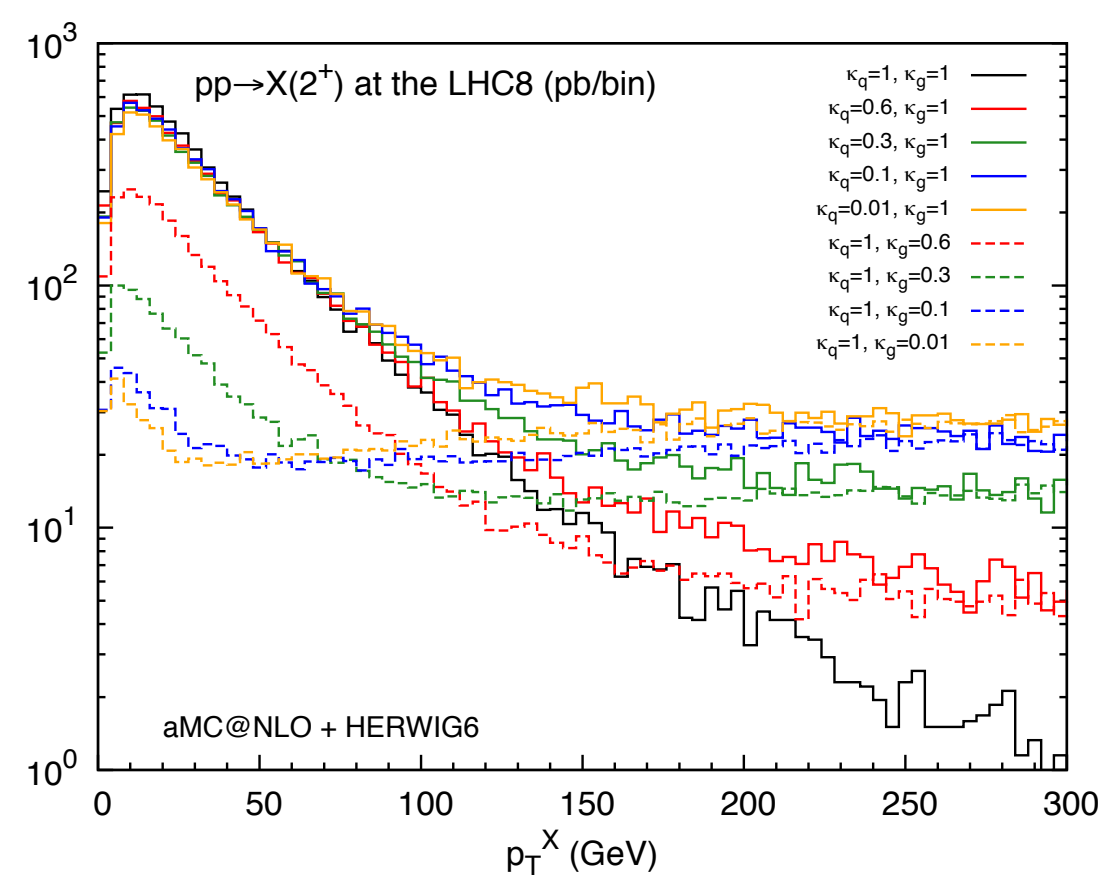

Figure 9. The transverse momentum $p_{T}^{X}$ of a spin-2 state with non universal couplings to quarks and gluons $\kappa_{q} \neq \kappa_{g}$ as obtained from AMC@NLO.

In this section, we address this question specifically for Higgs production. The case of spin-0 state is trivial; we have just used it in order to check that our observables are correctly defined. On the other hand, the spin-1 case is a possibly interesting one. However, the effects we aim at studying can hardly be seen in inclusive production, since $X_{1}$ is dominantly produced through the $q \bar{q}$ channel, and only the tiny mass of the initial state quarks and the virtuality of the gluon initiated quarks can generate the helicity-zero state. Some effects could be visible in subdominant production mechanisms, such as VBF, $V X_{1}$ or $t \bar{t} X_{1}$ associated production, but we shall not investigate them here. We are thus left with the case of a spin-2 particle, which we shall deal with in the following, by considering its $\gamma \gamma$ and $Z Z \rightarrow 4 \ell$ decay channels. We shall present results obtained with the ME+PS approach. As was the case of section 3, we have verified that AMC@NLO predictions are fairly close to those of ME+PS.

In order to introduce the argument in a simplified way, let us consider the production of a spin-2 boson in the universal coupling scenario, at the Born level (i.e. without any final-state partons), and in the partonic rest frame. In this way, the polarisation of $X_{2}$ lies along the beam axis and takes the values of $\pm 2( \pm 1)$ for the $g g$-channel $(q \bar{q}$-channel) contribution. The distributions in the decay angle $\theta^{*}$ can be expressed in terms of some $d$ functions, whose forms depend on the initial- and final-state particle helicities. The two different production modes lead to totally different $\theta^{*}$ distributions; specifically, one has:

$$
\begin{aligned}
\frac{d \sigma(g g)}{d \cos \theta^{*}} \propto\left|d_{22}^{2}\left(\theta^{*}\right)\right|^{2}+\left|d_{2-2}^{2}\left(\theta^{*}\right)\right|^{2} & =\frac{1}{8}\left(1+6 \cos ^{2} \theta^{*}+\cos ^{4} \theta^{*}\right), \\
\frac{d \sigma(q \bar{q})}{d \cos \theta^{*}} \propto\left|d_{12}^{2}\left(\theta^{*}\right)\right|^{2}+\left|d_{1-2}^{2}\left(\theta^{*}\right)\right|^{2} & =\frac{1}{2}\left(1-\cos ^{4} \theta^{*}\right) .
\end{aligned}
$$




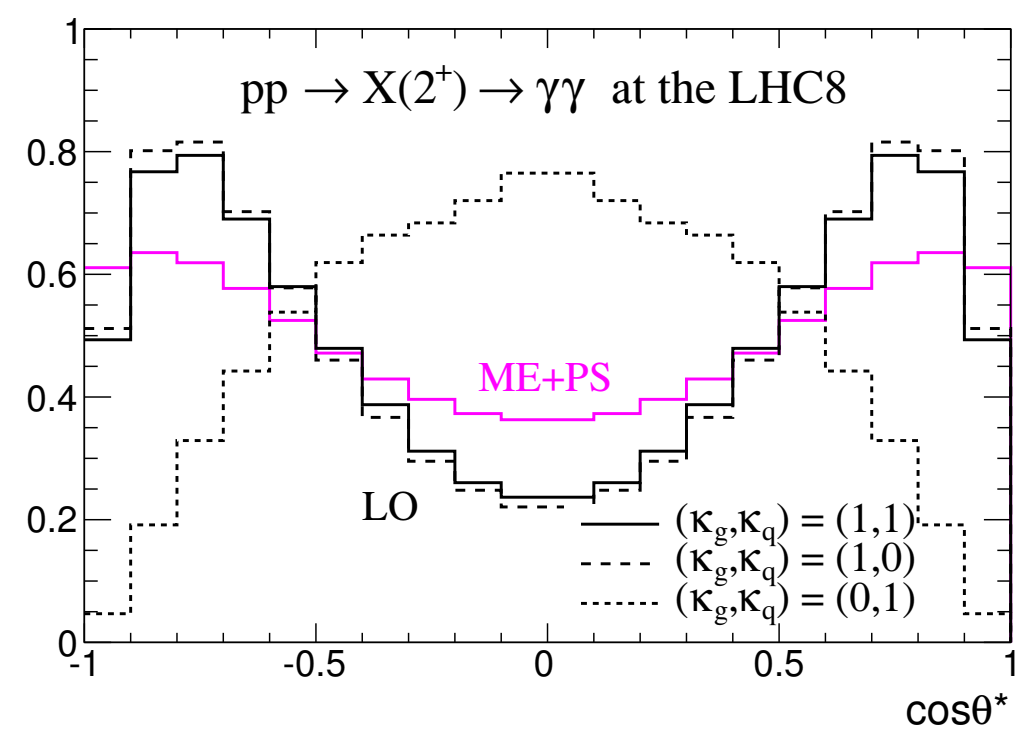

Figure 10. Normalised distribution of $p p \rightarrow X_{2} \rightarrow \gamma \gamma$ events with respect to $\cos \theta^{\star}$ resulting from different approaches: LO with $\left(\kappa_{g}, \kappa_{q}\right)=(1,1)$ (solid black line), LO with $\left(\kappa_{g}, \kappa_{q}\right)=(1,0)$ (dashed black line) LO with $\left(\kappa_{g}, \kappa_{q}\right)=(0,1)$ (dotted black line) and ME+PS merging approach with $\left(\kappa_{g}, \kappa_{q}\right)=(1,1)$ (solid magenta line).

The dominance of either the $g g$ or $q \bar{q}$ channels can be clearly seen in figure 10 - the former leading to enhanced cross sections at the end points $\left(\cos \theta^{*}= \pm 1\right.$ - however, right on the end points there is a kinematical-driven depletion), which are on the other hand associated with a suppressed production in the latter case. Unfortunately, the clarity of this picture is blurred by the inclusion of higher-order effects, which we present here only for the universal-coupling scenario. This is clearly the effect of the much richer helicity configurations of matrix elements with larger multiplicities, and of the more involved parton-luminosity structure at higher orders, whose role is therefore essential for proper phenomenological studies. ${ }^{5}$

In the case of the decay of $X_{2}$ to four leptons more observables can be studied. In figure 11 we show the distributions of the invariant masses of the two lepton pairs $m_{1}, m_{2}$ (with $m_{1}>m_{2}$ ), $\cos \theta^{*}, \cos \theta_{2}$, and $\phi_{1}$. While differences in the invariant mass distribution of the lepton pairs are minor, the angle distributions, and especially the $\cos \theta^{*}$ one, are affected by higher order corrections.

\subsection{Determination of the $C P$-mixing of a spin-0 state with the matrix element method}

In this section we illustrate how the availability of the Higgs Characterisation Model in FEynRules and MADGRaph 5 opens the way to using advanced analysis tools such as MADWEIGHT [107].

\footnotetext{
${ }^{5}$ Note that, in the presence of extra radiation, the angle $\theta^{*}$ is defined as the angle between the momentum of $X_{2}$ in the laboratory frame and that of the photon in the $X_{2}$ rest frame.
} 

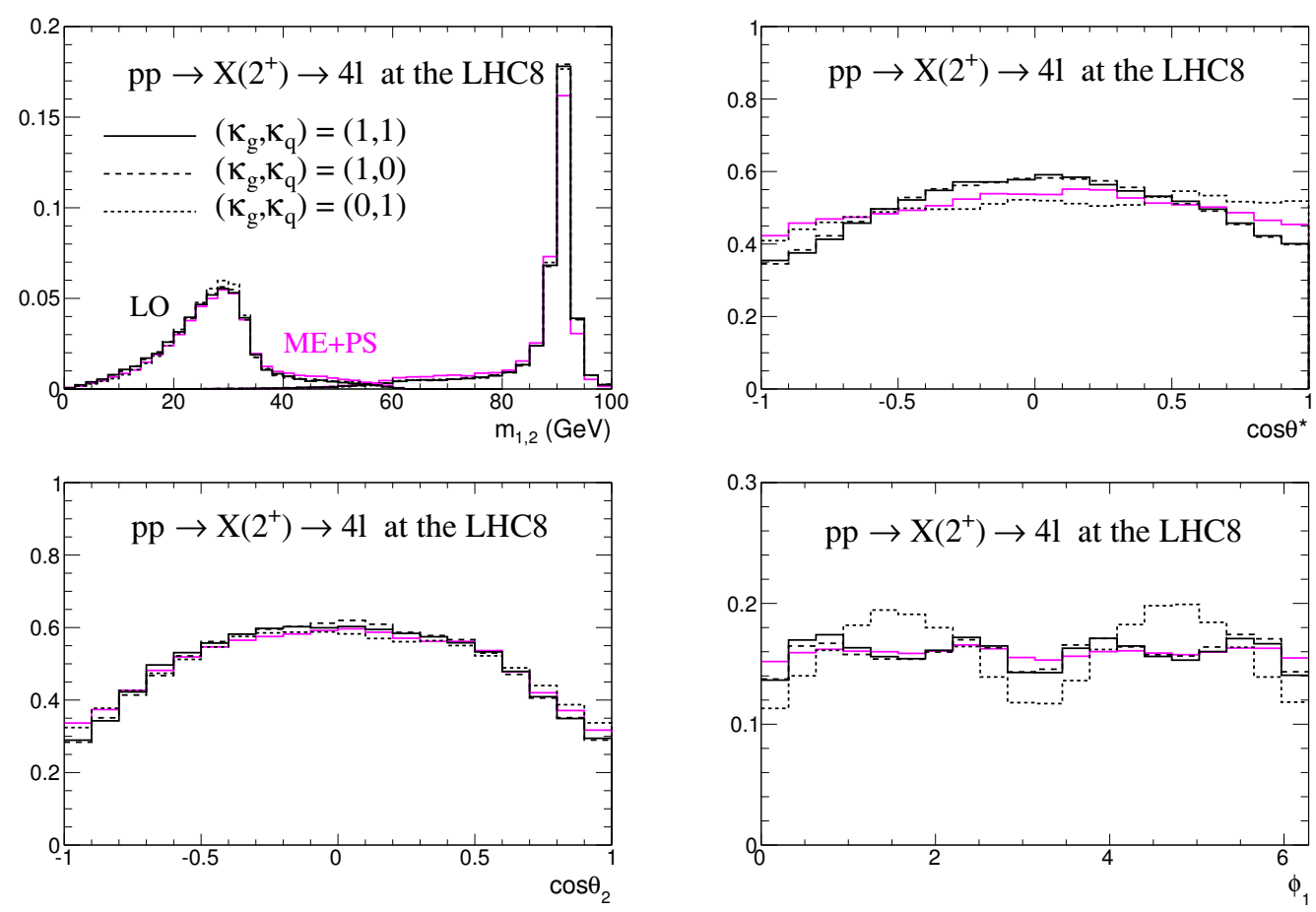

Figure 11. Normalised distributions in $p p \rightarrow X_{2} \rightarrow \mu^{+} \mu^{-} e^{+} e^{-}$at LO and in presence of extra QCD radiation as described by a ME+PS merged sample. The solid curve corresponds to the $\left(\kappa_{g}, \kappa_{q}\right)=(1,1)$ case.

The matrix element method (MEM) [108] has been successfully employed in the context of the Higgs boson discovery and spin determination [15, 16, 109-112]. Recently, the MEM has been used by both the ATLAS [6] and CMS [113] experiments to test the hypothesis of a SM-like scalar boson against other possible $J^{P}$ assignments. The CMS experiment has also considered the possibility that the coupling of the newly-discovered resonance to the $Z$ boson is a mixture of the $C P$-even operator $Z_{\mu} Z^{\mu}$ and the $C P$-odd operator $Z_{\mu \nu} \tilde{Z}^{\mu \nu}$.

We now show how, by using the MEM and its automatic implementation in MADWEIGHT, the analysis of the properties of the new resonance can be further extended by considering a specific example, namely the discrimination of a SM-like coupling to the $Z$ boson against the hypothesis of a coupling involving a superposition of the higher-dimension operators $Z_{\mu \nu} Z^{\mu \nu}\left(C P\right.$-even) and $Z_{\mu \nu} \tilde{Z}^{\mu \nu}$ (CP-odd) (see the fourth line of eq. (2.4)). For the sake of illustration, we consider here only a simplified analysis by: i) neglecting the presence of background events; ii) neglecting any resolution effects associated with the reconstruction of the leptons; and iii) considering only the channel $X_{0} \rightarrow \mu^{+} \mu^{-} e^{+} e^{-}$. We stress, however, that our approach and techniques are general enough to allow one to perform more complete studies, including background and resolution effects. Samples of events at $\sqrt{s}=8 \mathrm{TeV}$ are generated with the ME+PS approach that was presented in section 3. We select events where each of the four leptons satisfies $p_{T}>7 \mathrm{GeV}$ and $|\eta|<2.4$. We generate twelve samples of $3 \times 10^{4}$ events with different coupling parameters: the first sample is generated with $\kappa_{S M}=c_{\alpha}=1, \kappa_{H Z Z}=\kappa_{A Z Z}=0$, and corresponds to 

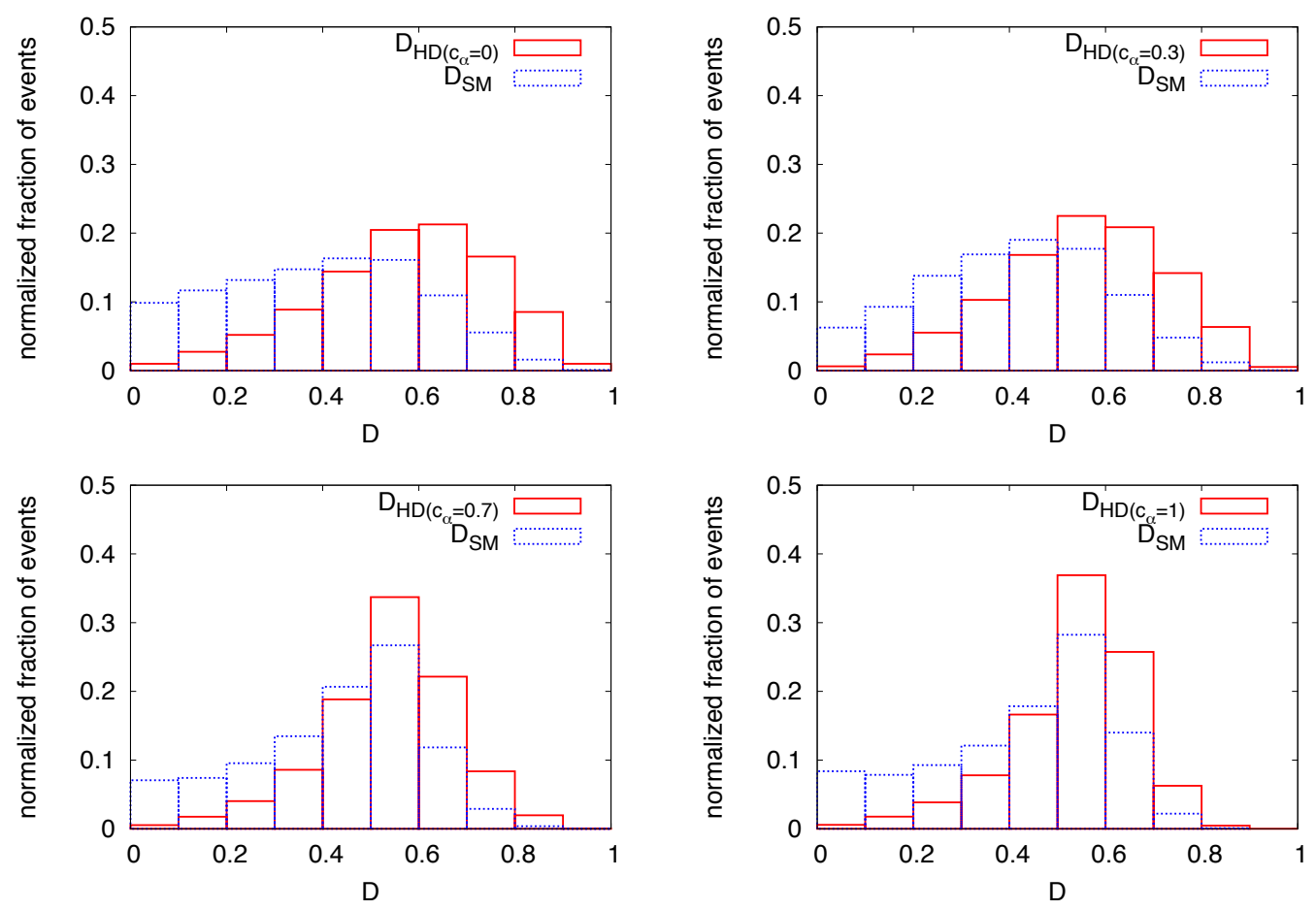

Figure 12. Normalised distributions per event with respect to the MEM-based discriminant $D$, for specific values of the mixing parameter of $c_{\alpha}: 0,0.3,0.7$ and 1.0 .

the SM (referred to as the SM hypothesis hereafter), whereas the eleven other samples are generated with $\kappa_{S M}=0, \kappa_{H Z Z}=\kappa_{A Z Z}=1$ and with $c_{\alpha}$ ranging from 0 to 1 in steps of 0.1. They correspond to the assumption that the yield originates from the contribution of higher-dimension operators with a parity-mixing parameter $c_{\alpha}$ (referred to as the $\operatorname{HD}\left(c_{\alpha}\right)$ hypothesis hereafter). All events in the twelve samples are passed to MADWEIGHT [107] for the automatic evaluation of the weights.

Following the approach of ref. [114], for a generic event $i$ with kinematics $\boldsymbol{x}_{i}$ the MEM-based observable $D_{i}$ for testing the SM against the $\operatorname{HD}\left(c_{\alpha}\right)$ hypotheses is evaluated as follows:

$$
D_{i}=\frac{P\left[\boldsymbol{x}_{i} \mid \operatorname{HD}\left(c_{\alpha}\right)\right]}{P\left[\boldsymbol{x}_{i} \mid \operatorname{HD}\left(c_{\alpha}\right)\right]+P\left[\boldsymbol{x}_{i} \mid \mathrm{SM}\right]} .
$$

Expected (normalised) distributions of $\mathrm{SM}$ and $\operatorname{HD}\left(c_{\alpha}\right)$ events in this observable are denoted by $D_{\mathrm{SM}}$ and $D_{\mathrm{HD}\left(c_{\alpha}\right)}$, and are shown in figure 12 for some specific values of $c_{\alpha}$.

In order to assess the significance that can be achieved at the LHC to reject the hypothesis $\operatorname{HD}\left(c_{\alpha}\right)$ if the $\mathrm{SM}$ hypothesis is realised, we consider a large number of pseudoexperiments, each with a given number $N$ of $X_{0} \rightarrow \mu^{+} \mu^{-} e^{+} e^{-}$events. We set $N=10$, which is close to the number of events (in the SM hypothesis) expected to be reconstructed in the ATLAS [6] and CMS [113] detectors at $\sqrt{s}=8 \mathrm{TeV}$, in this specific decay channel and with an integrated luminosity of $20 \mathrm{fb}^{-1}$. For each event, the corresponding $D_{i}$ value is generated according to the probability law $D_{\mathrm{SM}}$ (in the case of a SM pseudo- 

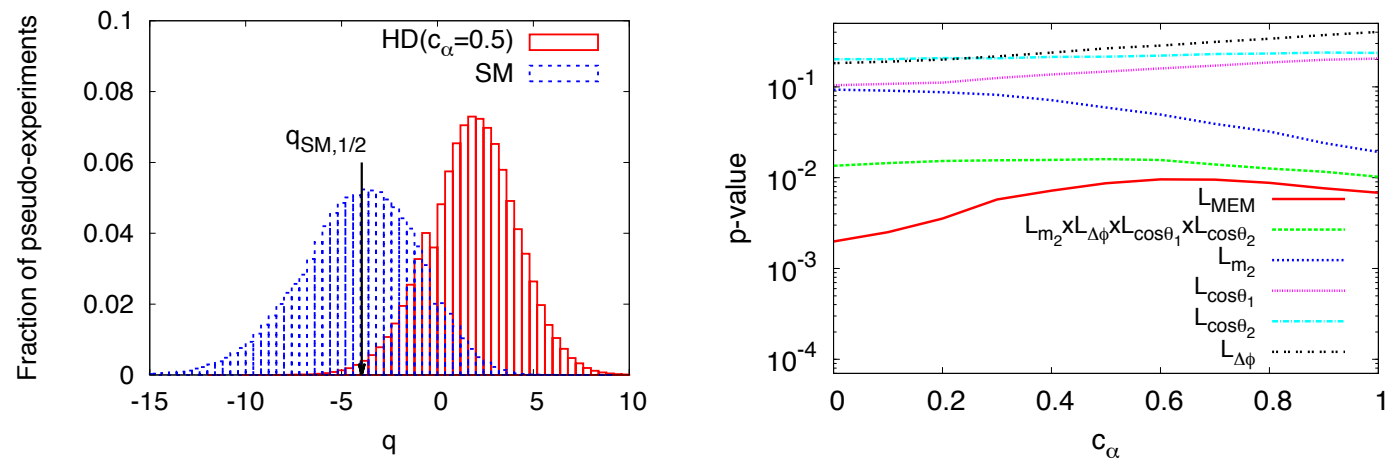

Figure 13. Left: distributions of pseudo-experiments with respect to $q=\ln \left(L_{\mathrm{MEM}}\right)$ for the case of $c_{\alpha}=0.5$. Right: expected $p$-value at which hypothesis $\operatorname{HD}\left(c_{\alpha}\right)$ is rejected if hypothesis $\mathrm{SM}$ is realised, as a function of $c_{\alpha}$ and for different choices of the likelihood function.

experiment) or $D_{\mathrm{HD}\left(c_{\alpha}\right)}$ (in the case of a $\operatorname{HD}\left(c_{\alpha}\right)$ pseudo-experiment) which are shown in figure 12. This procedure is used to generate $10^{6}$ pseudo-experiments under each hypothesis, $\mathrm{SM}$ or $\operatorname{HD}\left(c_{\alpha}\right)$.

For each pseudo-experiment the likelihood ratio $L$ is calculated as follows:

$$
L_{\mathrm{MEM}}=\prod_{i}^{N} \frac{P\left[\boldsymbol{x}_{i} \mid \mathrm{HD}\left(c_{\alpha}\right)\right]}{P\left[\boldsymbol{x}_{i} \mid \mathrm{SM}\right]}=\prod_{i}^{N} \frac{D_{i}}{1-D_{i}} .
$$

The resulting $\mathrm{SM}$ and $\operatorname{HD}\left(c_{\alpha}\right)$ distributions of pseudo-experiments in $q=\ln \left(L_{\mathrm{MEM}}\right)$ are shown in figure 13 (left) for the specific case of $c_{\alpha}=0.5$. The significance is estimated by calculating the median $q_{\mathrm{SM}, 1 / 2}$ of the SM distribution and by counting the fraction of pseudo-experiments in the $\operatorname{HD}\left(c_{\alpha}\right)$ distribution with $q<q_{\mathrm{SM}, 1 / 2}$. Such a fraction of events provides us with an estimate of the $p$-value associated with the statistical test for rejecting hypothesis $\operatorname{HD}\left(c_{\alpha}\right)$ if the $\mathrm{SM}$ hypothesis is realised. The $p$-value as a function of $c_{\alpha}$ is shown in figure 13 (right).

The power of the MEM can be illustrated by comparing the significance that is achieved when using the MEM-based likelihood function $L_{\mathrm{MEM}}$ with the significance resulting from a likelihood function built upon the cross section differential in the observable $\mathcal{O}$ :

$$
L_{\mathcal{O}}=\prod_{i}^{N} \frac{\sigma_{\mathrm{HD}\left(c_{\alpha}\right)}^{-1} \frac{d \sigma_{\mathrm{HD}\left(c_{\alpha}\right)}}{d \mathcal{O}}\left(\mathcal{O}_{i}\right)}{\sigma_{\mathrm{SM}}^{-1} \frac{d \sigma_{\mathrm{SM}}}{d \mathcal{O}}\left(\mathcal{O}_{i}\right)} .
$$

In this specific example, $\mathcal{O}$ is chosen in the set of spin/parity observables $\left\{m_{2}, \Delta \phi, \cos \theta_{1}\right.$, $\left.\cos \theta_{2}\right\}$ defined in ref. [16]. The discriminant power of each of these four variables taken separately can be assessed by using the same Monte Carlo procedure as before, with $L_{\text {MEM }}$ replaced by $L_{\mathcal{O}}$. The resulting $p$-values as a function of $c_{\alpha}$ are also shown in figure 13 (right). Even when the likelihood function is set equal to the product $L_{m_{2}} \times L_{\Delta \phi} \times L_{\cos \theta_{1}} \times L_{\cos \theta_{2}}$, one observes that the significance is smaller than the one obtained by the MEM-based likelihood analysis, presumably because all correlations among reconstructed variables can be kept only in the latter case. 


\section{Summary and outlook}

The determination of the properties and interactions of the newly-discovered boson will be one of the top priorities of the experimental and theory communities in the forthcoming years, through which a definite answer will be given to the question of whether this is, or is rather not, the Higgs boson predicted by the Standard Model.

In this paper, we have advocated the use of an effective-theory approach as a powerful way to tackle this and related issues. We have also shown how such an approach becomes an extremely flexible and multifaceted tool when its lagrangian is embedded into the FeynRules and MadGraph 5 frameworks (through what we have called the Higgs Characterisation model), owing to the capability of the latter to include higher-order QCD corrections, both at the tree-, multi-parton level (ME+PS) and with next-to-leading order accurate calculations (AMC@NLO) matched to parton showers. Indeed, we have found evidence of the fact that such corrections are a very important ingredient for performing sensible phenomenology studies.

In the spirit of an automated approach, we could only give here a glimpse of the possibilities of the Higgs Characterisation model, which can be fully exhausted only in the context of complete physics analyses such as those performed by the LHC experiments. In particular, we have restricted ourselves to the case of inclusive production, and have considered two directions. Firstly, we have validated our approach in different ways, prominent among which is the observation that the ME+PS and AMC@NLO results are fairly consistent with each other. Secondly, we have presented three sample applications, selected because they summarise well the flexibility and the potential for accuracy of our approach. In particular: a) We have shown that, in the case of the production of a spin- 2 state with non-universal couplings (i.e., the only spin-2 case still phenomenologically viable), leading-order simulations give vastly inadequate predictions for both rate and shapes, being in particular unable to account for a unitarity-violating behaviour at large transverse momenta. b) We have given examples of how higher-order QCD corrections can significantly affect spin-correlation variables that may help in the discrimination of a spin-2 state from other spin hypotheses. c) We have proven that the Higgs Characterisation model allows one to use effectively advanced analysis tools such as MADWEIGHT, by presenting a study on the determination of the amount of $C P$ mixing of a spin-0 resonance based on matrix-element methods.

Improvements or further developments of our framework could be achieved on two main directions. From the model point of view, we have built an effective lagrangian that is general enough to include all the effects coming from the (gauge-invariant) set of dimension-six operators that affect the three-point Higgs interactions, with exactly one Higgs particle. One could therefore complete the effective lagrangian to include the full set of operators which involve modifications or new four-point interactions, including those featuring two Higgs particles. This is straightforward and work in progress.

Regarding the possibility of accurate simulation we remark that the automation of the ME+PS techniques employed in this paper is complete, and thus such techniques can be used regardless of the process and/or applications one considers. On the other hand, the 
AMC@NLO predictions have been obtained by partly using analytically-computed virtual contributions, due to the present limitations in the calculations of one-loop matrix elements stemming from a user-defined lagrangian. While we remark that, for certain types of production mechanisms such as VBF or associated production, the current framework is already sufficient for automatic one-loop computations (owing to the structures of the virtuals in such production processes), we also point out that the outlook is quite positive, given the recent progress in FEYNRULES which will lift the limitations mentioned above. Among other things, this will also provide one with the possibility of using the FxFx NLO-merging [115] framework, which has the advantages of both the ME+PS and AMC@NLO approaches. Regardless of this near-future developments, it is important to keep in mind that the ME+PS and AMC@NLO results have complementary benefits, the former being better in those corners of the phase space which receive significant contributions from multi-leg matrix elements, while the latter being able to give realistic estimates of perturbative uncertainties.

\section{Acknowledgments}

In primis, we would like to thank all the members of Higgs Cross Section Working Group for the encouragement in pursuing the work presented here. We also thank Jean-Marc Gérard, Christophe Grojean, Kaoru Hagiwara, Gino Isidori, Maggie Müehlleitner, Riccardo Rattazzi, Veronica Sanz, and Reisaburo Tanaka for many stimulating discussions.

FM thanks KITP for hospitality during the last phases of this work. SF is on leave of absence from INFN, sezione di Genova. This work has been supported in part by the ERC grant 291377 "LHCtheory: Theoretical predictions and analyses of LHC physics: advancing the precision frontier", by the Forschungskredit der Universität Zürich, by the Swiss National Science Foundation (SNF) under contract 200020-138206 and by the Research Executive Agency (REA) of the European Union under the Grant Agreement number PITNGA-2010-264564 (LHCPhenoNet). This work has been realised in the framework of the BELSPO Belgium/India collaboration project HT\&LHC-BL/10/IN05. The work of FM, FD, MZ is supported by the IISN "MadGraph" convention 4.4511.10, the IISN "Fundamental interactions" convention 4.4517.08. PA is supported by a Marie Curie Intra-European Fellowship (PIEF-GA-2011-299999 PROBE4TeVSCALE). PdA and KM are supported in part by the Belgian Federal Science Policy Office through the Interuniversity Attraction Pole P7/37, in part by the "FWO-Vlaanderen" through the project G.0114.10N, and in part by the Strategic Research Program "High Energy Physics" and the Research Council of the Vrije Universiteit Brussel. SS is supported by the Senior Reach Fellowship of UGC, New Delhi. MKM and VR acknowledge the support from RECAPP@HRI.

\section{A Spin-1 hypothesis and two-photon final states}

In this appendix we comment on the possibility that a spin- 1 resonance $X_{1}$ might lead to a peak structure in the $\gamma \gamma$ invariant mass spectrum, as suggested, for example, in ref. [116]. 
The Landau-Yang theorem $[49,50]$ states that a spin-1 state cannot couple to two identical massless vectors. The theorem is based on Bose and Lorentz symmetry and assumes the massive spin- 1 state to be on-shell, i.e. to have only three degrees of freedom. The question that arises, though, is whether $g g \rightarrow X_{1} \rightarrow \gamma \gamma$ scattering might occur if the particle is off-shell and, were that the case, whether the interference with the background $g g \rightarrow \gamma \gamma$ via a box loop might still give rise to a structure in the invariant mass spectrum of the two photons around the $X_{1}$ mass.

To analyse this possibility one can proceed in different ways. To show that the question itself is relevant and to make our argument as simple and concrete as possible, we consider $g g \rightarrow Z^{(*)} \rightarrow \gamma \gamma$ scattering in the SM, where the $g g \rightarrow Z^{(*)}$ and $Z^{(*)} \rightarrow \gamma \gamma$ transitions happen via fermion triangle loops. Extending the results to the most general interactions with a generic vector state $X_{1}$ is straightforward.

The computation of the triangle loop is straightforward. First, only axial-vector coupling $\gamma^{\mu} \gamma_{5}$ between $Z$ and fermion line contributes, due to Furry's theorem ( $C$-invariance). Second, the diagrams are anomalous, yet the anomalies cancel in the SM once all the contributing fermions in a generation are considered (including therefore the charged lepton in the $\gamma \gamma$ case). In fact, the cancellation is exact and the result vanishes if the masses of the internal fermions are neglected, as normally done for the first and second generations. It is enough then to consider only the contributions from top, bottom and tau. The amplitude $Z \gamma \gamma$ via a $W$ loop is zero.

The $g\left(p_{1}\right) g\left(p_{2}\right) \rightarrow Z(P)$ vertex, taking place via a quark loop, can be computed solving the following integral

$$
\begin{aligned}
i \mathcal{V}_{g g \rightarrow Z}^{\mu \alpha \beta, a b}\left(p_{1}, p_{2}\right)= & \frac{8 \pi \alpha_{s} m_{W} I_{q}}{v \cos \theta_{W}} \\
& \times \delta^{a b} \int \frac{d^{4} k}{(2 \pi)^{4}} \frac{\operatorname{Tr}\left[\gamma^{\mu} \gamma_{5}\left(\not k+m_{q}\right) \gamma^{\alpha}\left(\not k+\not p_{1}+m_{q}\right) \gamma^{\beta}\left(\not k+\not p_{1}+\not p_{2}+m_{q}\right)\right]}{\left[k^{2}-m_{q}^{2}\right]\left[\left(k+p_{1}\right)^{2}-m_{q}^{2}\right]\left[\left(k+p_{1}+p_{2}\right)^{2}-m_{q}^{2}\right]} .
\end{aligned}
$$

We keep full dependence on the mass of the quark and we put the incoming gluons on their mass shell. Our result, in agreement with the result in appendix A of ref. [117], is

$$
i \mathcal{V}_{g g \rightarrow Z}^{\mu \alpha \beta, a b}\left(p_{1}, p_{2}\right)=\delta^{a b} \frac{\alpha_{s} m_{W} I_{q}}{2 \pi v \cos \theta_{W}}\left[1-\frac{4 m_{q}^{2}}{s} f\left(\frac{4 m_{q}^{2}}{s}\right)\right] \epsilon^{\alpha \beta \rho \sigma} \frac{2 p_{1 \rho} p_{2 \sigma}}{s} P^{\mu}
$$

where

$$
f(x)= \begin{cases}{\left[\arcsin \left(\frac{1}{\sqrt{x}}\right)\right]^{2}} & \text { if } \quad x \geq 1 \\ -\frac{1}{4}\left[\ln \left(\frac{1+\sqrt{1-x}}{1-\sqrt{1-x}}\right)-i \pi\right]^{2} & \text { if } \quad x<1\end{cases}
$$

Since $I_{t}=-I_{b}$, it is evident from eq. (A.2) that if the fermions in an isospin doublet have the same mass, their contributions sum to zero. Thus, as we previously stated, only top and bottom quarks give a non-negligible contribution to the vertex, and it effectively depends on $m_{t}^{2}-m_{b}^{2}$. The expression for the $Z \rightarrow \gamma \gamma$ vertex is analogous, including also the tau loop. 
The most important feature of eq. (A.2) is that the effective vertex is proportional to the $Z$ momentum $P^{\mu}$. The calculation of the $g g \rightarrow Z \rightarrow \gamma \gamma$ amplitude therefore entails a contribution of the type

$$
P^{\mu} \Pi_{\mu \nu} P^{\nu}
$$

where $\Pi_{\mu \nu}=-g_{\mu \nu}+\frac{P_{\mu} P_{\nu}}{m_{Z}^{2}}$ is the numerator of the $Z$ propagator in the unitary gauge. If we contract one of the two vertices, say the one with $P^{\mu}$, with the projector $\Pi_{\mu \nu}$ we find an expression proportional to

$$
\left(s-m_{Z}^{2}\right) P_{\nu}
$$

where $s=P^{2}$ is the usual Mandelstam variable. This entails that the amplitude squared for the on-shell decay (or production) $Z \rightarrow g g$ or $Z \rightarrow \gamma \gamma$ is zero, in agreement with the Landau-Yang theorem. ${ }^{6}$

When, instead, one wants to compute the transition amplitude $g g \rightarrow Z \rightarrow \gamma \gamma$, one has to pay attention, because a blind application of Feynman rules leads to a wrong result. To illustrate this, we now proceed in the same way as in ref. [116]. Once contracted with $P^{\mu} P^{\nu}$, the $Z$ propagator in the unitary gauge gives

$$
\frac{i s}{m_{Z}^{2}} \frac{s-m_{Z}^{2}}{s-m_{Z}^{2}+i \Gamma_{Z} m_{Z}} \text {. }
$$

This expression complies with the Landau-Yang theorem, as it is zero when the $Z$ is onshell; however, it also displays a non-trivial structure at $s=m_{Z}^{2}$, i.e. a dip. It is then natural to wonder whether such contribution might lead to a peak or a dip-peak structure at $s=m_{Z}^{2}$ when interfered with the $g g \rightarrow \gamma \gamma$ continuous background, as suggested in ref. [116]. An explicit calculation, which we do not report here, shows that this is indeed the case.

However, the derivation above is not correct, as it relies on having put a non-zero width for the $Z$ in the propagator not in a consistent way. ${ }^{7}$

In fact, there is no pole at $s=m_{Z}^{2}$ in the $g g \rightarrow Z \rightarrow \gamma \gamma$ amplitude, as numerator and denominator exactly cancel when we use the propagator

$$
P^{\mu} \frac{i \Pi_{\mu \nu}}{s-m_{Z}^{2}} P^{\nu}=\frac{i s}{m_{Z}^{2}}
$$

and there is no need to introduce a width in the denominator in the first place. The same result can be obtained by introducing the width in a consistent way, i.e., using the complex mass scheme and replacing $m_{Z}^{2} \rightarrow m_{Z}^{2}-i \Gamma_{Z} m_{Z}$ everywhere in the expression of the amplitude.

\footnotetext{
${ }^{6}$ The original theorem holds only for the decay of a massive spin-1 state to two photons; since, however, the $Z$ colour structure is trivial, the same theorems is valid in this particular case also for the decay to two gluons.

${ }^{7}$ We thank Kaoru Hagiwara for enlightening discussions on this point.
} 
The results for the helicity amplitudes of the process therefore read

$$
\mathcal{M}_{--++}^{g g \rightarrow Z \rightarrow \gamma \gamma}=S_{--++} \delta^{a b} \mathcal{M}_{g g \rightarrow Z} \frac{s}{m_{Z}^{2}} \mathcal{M}_{Z \rightarrow \gamma \gamma}
$$

and

$$
\mathcal{M}_{++++}^{g g \rightarrow Z \rightarrow \gamma}=S_{++++} \delta^{a b} \mathcal{M}_{g g \rightarrow Z} \frac{s+2 t}{m_{Z}^{2}} \mathcal{M}_{Z \rightarrow \gamma \gamma},
$$

where $S_{ \pm \pm++}$are spinor phases and

$$
\begin{aligned}
& \mathcal{M}_{g g \rightarrow Z}=\frac{\alpha_{s} m_{W}}{2 \pi v \cos \theta_{W}} \sum_{q=t, b} I_{q}\left[1-\frac{4 m_{q}^{2}}{s} f\left(\frac{4 m_{q}^{2}}{s}\right)\right], \\
& \mathcal{M}_{Z \rightarrow \gamma \gamma}=\frac{\alpha m_{W}}{\pi v \cos \theta_{W}} \sum_{f=t, b, \tau} N_{c}^{(f)} Q_{f}^{2} I_{f}\left[1-\frac{4 m_{f}^{2}}{s} f\left(\frac{4 m_{f}^{2}}{s}\right)\right] .
\end{aligned}
$$

Such amplitudes do not display any enhancement or zero at the $Z$ pole and therefore cannot lead to any peak or dip-peak structure in the $\gamma \gamma$ invariant mass spectrum around the $Z$ mass.

It is interesting, however, to note that the amplitudes are not zero and can be interpreted as coming from a contact $g g \gamma \gamma$ interaction. An analogous result can be obtained, for example, calculating the amplitude $g g \rightarrow Z \rightarrow t \bar{t}$ which is also non-vanishing, proportional to $m_{t}\left(m_{t}^{2}-m_{b}^{2}\right)$ and without any structure at $s=m_{Z}^{2}$.

It is easy to see that any possible effective vertex that can be written for a generic vector $X_{1}$ and two massless identical gauge vectors, $g g$ or $\gamma \gamma$, due to the Landau-Yang theorem either gives a vanishing contribution to $g g \rightarrow X_{1} \rightarrow \gamma \gamma$ or leads to the cancellation of the propagator, effectively leaving a $g g \rightarrow \gamma \gamma$ contact interaction.

For example, an expression analogous to the SM one for the $g g \rightarrow Z$ vertex can be deduced from the dimension-six operator

$$
\mathcal{L}_{g g Z}=\frac{1}{\Lambda^{2}}\left(\partial_{\mu} Z^{\mu}\right) G_{\alpha \beta}^{a} \tilde{G}^{a, \alpha \beta},
$$

which makes manifest that the non-vanishing result for the amplitude is due to the nonconservation of the neutral axial-current in the SM due to the fermion masses.

\section{B Divergences in the $p p \rightarrow X_{2}$ computation at NLO}

As mentioned in section 4.1, when $\kappa_{q}=\kappa_{g}$ all the UV divergences present at the intermediate stages of an NLO calculation cancel with the standard counterterms, thanks to the fact that the E-M tensor is conserved. When $\kappa_{q} \neq \kappa_{g}$ such cancellations are not there any longer, and the two couplings need to be renormalised. In this appendix we illustrate how the renormalisation is performed in this case.

One starts from the renormalisation mixing matrix $Z_{i j}$ with $i, j=q, \bar{q}, g$, which can be easily computed from the quark and gluon contributions to $T_{\mu \nu}^{q, g}$ at one loop. These contributions are both UV and IR divergent; studying the UV behaviour of such contributions, 
one can obtain the renormalisation matrix $Z_{i j}$. Defining

$$
Z_{i j}=1+\frac{\alpha_{s}\left(\mu_{R}^{2}\right)}{4 \pi} \frac{Z_{i j}^{(1)}}{\epsilon}
$$

one obtains

$$
Z_{q q}^{(1)}=-\frac{16}{3} C_{F}, \quad Z_{q g}^{(1)}=\frac{16}{3} C_{F}, \quad Z_{g g}^{(1)}=-\frac{8}{3} n_{f} T_{F}, \quad Z_{g q}^{(1)}=\frac{8}{3} n_{f} T_{F} .
$$

From $Z_{i j}$ it is possible to obtain the overall renormalisation constant for the operator $T_{\mu \nu}^{q}+T_{\mu \nu}^{g}$, which, as expected, is the identity. Due to the operator mixing at $\mathcal{O}\left(\alpha_{s}\right)$ correction, the couplings $\kappa_{q, g}$ develop a scale dependence. The coupled renormalisation group equations are controlled by the anomalous dimension matrix defined by

$$
\gamma=Z^{-1} \mu_{R}^{2} \frac{d Z}{d \mu_{R}^{2}},
$$

and hence these couplings run with the scale $\mu_{R}$.

The information on the $Z_{i j}$ matrix can be exploited to compute the NLO corrections to the $p p \rightarrow X_{2}$ process. The loop contributions will have diagrams in which the spin-2 state couples both to quarks and gluons. In the case in which $\kappa_{q} \neq \kappa_{g}$, UV divergences can be renormalised by using $Z_{i j}$. After renormalisation, as expected, the resulting expressions contain only IR divergences and finite terms. The IR divergences are proportional to $\kappa_{q}^{2}$ and $\kappa_{g}^{2}$ separately (no $\kappa_{q} \kappa_{g}$ terms), having double and single poles in $\epsilon(D=4+\epsilon$ is the space time dimension in dimensional regularisation). This confirms the universality of soft and collinear singularities of the virtual amplitudes. We find that the double and single pole terms contain the appropriate universal coefficients to cancel against those coming from real emission processes and mass factorisation counterterms hence providing a check of our computation with $\kappa_{q} \neq \kappa_{g}$.

Open Access. This article is distributed under the terms of the Creative Commons Attribution License which permits any use, distribution and reproduction in any medium, provided the original author(s) and source are credited.

\section{References}

[1] ATLAS collaboration, Observation of a new particle in the search for the Standard Model Higgs boson with the ATLAS detector at the LHC, Phys. Lett. B 716 (2012) 1 [arXiv:1207.7214] [INSPIRE].

[2] CMS collaboration, Observation of a new boson at a mass of $125 \mathrm{GeV}$ with the CMS experiment at the LHC, Phys. Lett. B 716 (2012) 30 [arXiv:1207.7235] [INSPIRE].

[3] ATLAS collaboration, Search for the Standard Model Higgs boson in the $H \rightarrow Z \gamma$ decay mode with pp collisions at $\sqrt{s}=7$ and $8 \mathrm{TeV}$, ATLAS-CONF-2013-009 (2013).

[4] ATLAS collaboration, Search for a Standard Model Higgs boson in $H \rightarrow \mu \mu$ decays with the ATLAS detector, ATLAS-CONF-2013-010 (2013). 
[5] ATLAS collaboration, Measurements of the properties of the Higgs-like boson in the two photon decay channel with the ATLAS detector using $25 \mathrm{fb}^{1}$ of proton-proton collision data, ATLAS-CONF-2013-012 (2013).

[6] ATLAS collaboration, Measurements of the properties of the Higgs-like boson in the four lepton decay channel with the ATLAS detector using $25 \mathrm{fb}^{1}$ of proton-proton collision data, ATLAS-CONF-2013-013 (2013).

[7] CMS collaboration, Evidence for a particle decaying to $W+W-$ in the fully leptonic final state in a standard model Higgs boson search in pp collisions at the LHC, CMS-HIG-13-003 (2013).

[8] CMS collaboration, Search for the Standard-Model Higgs boson decaying to tau pairs in proton-proton collisions at $\sqrt{s}=7$ and $8 \mathrm{TeV}$, CMS-HIG-13-004 (2013).

[9] CMS collaboration, Search for the standard model Higgs boson in the $Z$ boson plus a photon channel in pp collisions at $\sqrt{s}=7$ and 8 TeV, CMS-HIG-13-006 (2013).

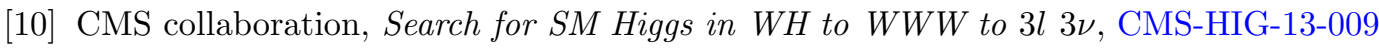
(2013).

[11] S. Weinberg, Implications of Dynamical Symmetry Breaking, Phys. Rev. D 13 (1976) 974 [INSPIRE].

[12] F. Englert and R. Brout, Broken Symmetry and the Mass of Gauge Vector Mesons, Phys. Rev. Lett. 13 (1964) 321 [INSPIRE].

[13] P.W. Higgs, Broken Symmetries and the Masses of Gauge Bosons, Phys. Rev. Lett. 13 (1964) 508 [inSPIRE].

[14] S. Choi, M. Muhlleitner and P. Zerwas, Theoretical Basis of Higgs-Spin Analysis in $H \rightarrow \gamma \gamma$ and $Z \gamma$ Decays, Phys. Lett. B 718 (2013) 1031 [arXiv:1209.5268] [InSPIRE].

[15] Y. Gao et al., Spin determination of single-produced resonances at hadron colliders, Phys. Rev. D 81 (2010) 075022 [arXiv: 1001.3396] [INSPIRE].

[16] S. Bolognesi et al., On the spin and parity of a single-produced resonance at the LHC, Phys. Rev. D 86 (2012) 095031 [arXiv: 1208.4018] [INSPIRE].

[17] J.S. Gainer, J. Lykken, K.T. Matchev, S. Mrenna and M. Park, Spherical Parametrization of the Higgs Boson Candidate, Phys. Rev. Lett. 111 (2013) 041801 [arXiv:1304.4936] [INSPIRE].

[18] K. Hagiwara, R. Szalapski and D. Zeppenfeld, Anomalous Higgs boson production and decay, Phys. Lett. B 318 (1993) 155 [hep-ph/9308347] [INSPIRE].

[19] G. Giudice, C. Grojean, A. Pomarol and R. Rattazzi, The strongly-interacting light Higgs, JHEP 06 (2007) 045 [hep-ph/0703164] [InSPIRE].

[20] B. Gripaios, A. Pomarol, F. Riva and J. Serra, Beyond the minimal composite Higgs model, JHEP 04 (2009) 070 [arXiv:0902.1483] [INSPIRE].

[21] I. Low, R. Rattazzi and A. Vichi, Theoretical constraints on the Higgs effective couplings, JHEP 04 (2010) 126 [arXiv:0907.5413] [inSPIRE].

[22] D.E. Morrissey, T. Plehn and T.M. Tait, Physics searches at the LHC, Phys. Rept. 515 (2012) 1 [arXiv:0912.3259] [INSPIRE].

[23] R. Contino, C. Grojean, M. Moretti, F. Piccinini and R. Rattazzi, Strong double Higgs production at the LHC, JHEP 05 (2010) 089 [arXiv:1002.1011] [INSPIRE]. 
[24] J. Espinosa, C. Grojean and M. Muhlleitner, Composite Higgs search at the LHC, JHEP 05 (2010) 065 [arXiv: 1003.3251] [INSPIRE].

[25] A. Azatov, R. Contino and J. Galloway, Model-independent bounds on a light Higgs, JHEP 04 (2012) 127 [Erratum ibid. 1304 (2013) 140] [arXiv: 1202.3415] [INSPIRE].

[26] J. Espinosa, C. Grojean, M. Muhlleitner and M. Trott, Fingerprinting Higgs suspects at the LHC, JHEP 05 (2012) 097 [arXiv: 1202.3697] [INSPIRE].

[27] J. Ellis and T. You, Global analysis of experimental constraints on a possible Higgs-like particle with mass $125 \mathrm{GeV}$, JHEP 06 (2012) 140 [arXiv:1204.0464] [INSPIRE].

[28] I. Low, J. Lykken and G. Shaughnessy, Have We Observed the Higgs (Imposter)?, Phys. Rev. D 86 (2012) 093012 [arXiv: 1207.1093] [InSPIRE].

[29] M. Montull and F. Riva, Higgs discovery: the beginning or the end of natural EWSB?, JHEP 11 (2012) 018 [arXiv:1207.1716] [InSPIRE].

[30] J. Espinosa, C. Grojean, M. Muhlleitner and M. Trott, First glimpses at Higgs' face, JHEP 12 (2012) 045 [arXiv: 1207.1717] [INSPIRE].

[31] D. Carmi, A. Falkowski, E. Kuflik, T. Volansky and J. Zupan, Higgs after the discovery: a status report, JHEP 10 (2012) 196 [arXiv:1207.1718] [INSPIRE].

[32] T. Corbett, O. Eboli, J. Gonzalez-Fraile and M. Gonzalez-Garcia, Constraining anomalous Higgs interactions, Phys. Rev. D 86 (2012) 075013 [arXiv:1207.1344] [InSPIRE].

[33] J. Ellis and T. You, Global analysis of the Higgs candidate with mass $125 \mathrm{GeV}$, JHEP 09 (2012) 123 [arXiv: 1207.1693] [INSPIRE].

[34] G. Passarino, NLO Inspired Effective Lagrangians for Higgs Physics, Nucl. Phys. B 868 (2013) 416 [arXiv: 1209.5538] [INSPIRE].

[35] T. Corbett, O. Eboli, J. Gonzalez-Fraile and M. Gonzalez-Garcia, Robust Determination of the Higgs Couplings: Power to the Data, Phys. Rev. D 87 (2013) 015022 [arXiv:1211.4580] [INSPIRE].

[36] K. Cheung, J.S. Lee and P.-Y. Tseng, Higgs Precision (Higgcision) Era begins, JHEP 05 (2013) 134 [arXiv:1302.3794] [INSPIRE].

[37] A. Falkowski, F. Riva and A. Urbano, Higgs At Last, arXiv:1303.1812 [INSPIRE].

[38] R. Contino, M. Ghezzi, C. Grojean, M. Muhlleitner and M. Spira, Effective Lagrangian for a light Higgs-like scalar, JHEP 07 (2013) 035 [arXiv:1303.3876] [INSPIRE].

[39] W. Buchmüller and D. Wyler, Effective Lagrangian Analysis of New Interactions and Flavor Conservation, Nucl. Phys. B 268 (1986) 621 [INSPIRE].

[40] B. Grzadkowski, M. Iskrzynski, M. Misiak and J. Rosiek, Dimension-six terms in the standard model Lagrangian, JHEP 10 (2010) 085 [arXiv: 1008.4884] [INSPIRE].

[41] N.D. Christensen et al., A comprehensive approach to new physics simulations, Eur. Phys. J. C 71 (2011) 1541 [arXiv:0906.2474] [INSPIRE].

[42] N.D. Christensen and C. Duhr, FeynRules - Feynman rules made easy, Comput. Phys. Commun. 180 (2009) 1614 [arXiv:0806.4194] [INSPIRE].

[43] C. Englert, D. Goncalves-Netto, K. Mawatari and T. Plehn, Higgs quantum numbers in weak boson fusion, JHEP 01 (2013) 148 [arXiv:1212.0843] [INSPIRE].

[44] C. Degrande et al., UFO - The Universal FeynRules Output, Comput. Phys. Commun. 183 (2012) 1201 [arXiv:1108.2040] [INSPIRE]. 
[45] N. Christensen, C. Degrande, C. Duhr and B. Fuks, Feynrules model database, http://feynrules.irmp.ucl.ac.be.

[46] G.C. Branco, L. Lavour and J.P. Silva, CP violation, Oxford University Press, Oxford U.K. (1999).

[47] K. Hagiwara, R. Peccei, D. Zeppenfeld and K. Hikasa, Probing the Weak Boson Sector in $e^{+} e^{-} \rightarrow W^{+} W^{-}$, Nucl. Phys. B 282 (1987) 253 [inSPIRE].

[48] W.-Y. Keung, I. Low and J. Shu, Landau-Yang Theorem and Decays of a Z' Boson into Two Z Bosons, Phys. Rev. Lett. 101 (2008) 091802 [arXiv:0806.2864] [inSPIRE].

[49] L. Landau, On the angular momentum of a two-photon system, Dokl. Akad. Nauk Ser. Fiz. 60 (1948) 207.

[50] C.-N. Yang, Selection Rules for the Dematerialization of a Particle Into Two Photons, Phys. Rev. 77 (1950) 242 [INSPIRE].

[51] G.F. Giudice, R. Rattazzi and J.D. Wells, Quantum gravity and extra dimensions at high-energy colliders, Nucl. Phys. B 544 (1999) 3 [hep-ph/9811291] [INSPIRE].

[52] T. Han, J.D. Lykken and R.-J. Zhang, On Kaluza-Klein states from large extra dimensions, Phys. Rev. D 59 (1999) 105006 [hep-ph/9811350] [INSPIRE].

[53] J. Ellis, R. Fok, D.S. Hwang, V. Sanz and T. You, Distinguishing 'Higgs' spin hypotheses using $\gamma \gamma$ and $W W^{*}$ decays, Eur. Phys. J. C 73 (2013) 2488 [arXiv:1210.5229] [InSPIRE].

[54] K. Hagiwara, J. Kanzaki, Q. Li and K. Mawatari, HELAS and MadGraph/MadEvent with spin-2 particles, Eur. Phys. J. C 56 (2008) 435 [arXiv:0805.2554] [inSPIRE].

[55] L. Randall and R. Sundrum, A large mass hierarchy from a small extra dimension, Phys. Rev. Lett. 83 (1999) 3370 [hep-ph/9905221] [INSPIRE].

[56] J. Alwall, M. Herquet, F. Maltoni, O. Mattelaer and T. Stelzer, MadGraph 5: going beyond, JHEP 06 (2011) 128 [arXiv:1106.0522] [INSPIRE].

[57] P. de Aquino, W. Link, F. Maltoni, O. Mattelaer and T. Stelzer, ALOHA: Automatic Libraries Of Helicity Amplitudes for Feynman Diagram Computations, Comput. Phys. Commun. 183 (2012) 2254 [arXiv:1108.2041] [InSPIRE].

[58] S. Frixione and B.R. Webber, Matching NLO QCD computations and parton shower simulations, JHEP 06 (2002) 029 [hep-ph/0204244] [INSPIRE].

[59] G. Isidori, A.V. Manohar and M. Trott, Probing the nature of the Higgs-like Boson via $h \rightarrow V F$ decays, arXiv:1305.0663 [INSPIRE].

[60] B. Grinstein, C.W. Murphy and D. Pirtskhalava, Searching for new physics in the three-body decays of the Higgs-like particle, JHEP 10 (2013) 077 [arXiv:1305.6938] [INSPIRE].

[61] G. Corcella et al., HERWIG 6: An Event generator for hadron emission reactions with interfering gluons (including supersymmetric processes), JHEP 01 (2001) 010 [hep-ph/0011363] [INSPIRE].

[62] M. Bahr et al., HERWIG++ physics and manual, Eur. Phys. J. C 58 (2008) 639 [arXiv:0803.0883] [INSPIRE].

[63] T. Sjöstrand, S. Mrenna and P.Z. Skands, PYTHIA 6.4 physics and manual, JHEP 05 (2006) 026 [hep-ph/0603175] [inSPIRE].

[64] T. Sjöstrand, S. Mrenna and P.Z. Skands, A Brief Introduction to PYTHIA 8.1, Comput. Phys. Commun. 178 (2008) 852 [arXiv:0710.3820] [INSPIRE]. 
[65] M.L. Mangano, M. Moretti and R. Pittau, Multijet matrix elements and shower evolution in hadronic collisions: $W b \bar{b}+n$ jets as a case study, Nucl. Phys. B 632 (2002) 343 [hep-ph/0108069] [INSPIRE].

[66] J. Alwall et al., Comparative study of various algorithms for the merging of parton showers and matrix elements in hadronic collisions, Eur. Phys. J. C 53 (2008) 473 [arXiv:0706.2569] [INSPIRE].

[67] J. Alwall, S. de Visscher and F. Maltoni, QCD radiation in the production of heavy colored particles at the LHC, JHEP 02 (2009) 017 [arXiv: 0810.5350] [INSPIRE].

[68] J. Alwall, Q. Li and F. Maltoni, Matched predictions for Higgs production via heavy-quark loops in the SM and beyond, Phys. Rev. D 85 (2012) 014031 [arXiv:1110.1728] [INSPIRE].

[69] R. Frederix, S. Frixione, F. Maltoni and T. Stelzer, Automation of next-to-leading order computations in QCD: the FKS subtraction, JHEP 10 (2009) 003 [arXiv:0908.4272] [INSPIRE].

[70] S. Frixione, Z. Kunszt and A. Signer, Three jet cross-sections to next-to-leading order, Nucl. Phys. B 467 (1996) 399 [hep-ph/9512328] [INSPIRE].

[71] S. Frixione, A general approach to jet cross-sections in QCD, Nucl. Phys. B 507 (1997) 295 [hep-ph/9706545] [INSPIRE].

[72] V. Hirschi et al., Automation of one-loop QCD corrections, JHEP 05 (2011) 044 [arXiv:1103.0621] [INSPIRE].

[73] G. Ossola, C.G. Papadopoulos and R. Pittau, Reducing full one-loop amplitudes to scalar integrals at the integrand level, Nucl. Phys. B 763 (2007) 147 [hep-ph/0609007] [InSPIRE].

[74] G. Ossola, C.G. Papadopoulos and R. Pittau, CutTools: a program implementing the OPP reduction method to compute one-loop amplitudes, JHEP 03 (2008) 042 [arXiv:0711.3596] [INSPIRE].

[75] P. Draggiotis, M. Garzelli, C. Papadopoulos and R. Pittau, Feynman rules for the rational part of the QCD 1-loop amplitudes, JHEP 04 (2009) 072 [arXiv: 0903.0356] [INSPIRE].

[76] M. Garzelli, I. Malamos and R. Pittau, Feynman rules for the rational part of the Electroweak 1-loop amplitudes, JHEP 01 (2010) 040 [Erratum ibid. 1010 (2010) 097] [arXiv:0910.3130] [INSPIRE].

[77] M. Garzelli, I. Malamos and R. Pittau, Feynman rules for the rational part of the Electroweak 1-loop amplitudes in the $R_{\xi}$ gauge and in the Unitary gauge, JHEP 01 (2011) 029 [arXiv: 1009.4302] [INSPIRE].

[78] H.-S. Shao, Y.-J. Zhang and K.-T. Chao, Feynman Rules for the Rational Part of the Standard Model One-loop Amplitudes in the 't Hooft-Veltman $\gamma_{5}$ Scheme, JHEP 09 (2011) 048 [arXiv: 1106.5030] [INSPIRE].

[79] H.-S. Shao and Y.-J. Zhang, Feynman rules for the rational part of one-loop QCD corrections in the MSSM, JHEP 06 (2012) 112 [arXiv:1205.1273] [INSPIRE].

[80] S. Dawson, Radiative corrections to Higgs boson production, Nucl. Phys. B 359 (1991) 283 [INSPIRE].

[81] R.P. Kauffman and W. Schaffer, QCD corrections to production of Higgs pseudoscalars, Phys. Rev. D 49 (1994) 551 [hep-ph/9305279] [INSPIRE].

[82] P. Mathews, V. Ravindran and K. Sridhar, NLO-QCD corrections to dilepton production in the Randall-Sundrum model, JHEP 10 (2005) 031 [hep-ph/0506158] [INSPIRE]. 
[83] M. Kumar, P. Mathews, V. Ravindran and A. Tripathi, Diphoton signals in theories with large extra dimensions to NLO QCD at hadron colliders, Phys. Lett. B 672 (2009) 45 [arXiv:0811.1670] [INSPIRE].

[84] M. Kumar, P. Mathews, V. Ravindran and A. Tripathi, Direct photon pair production at the LHC to order $\alpha_{s}$ in TeV scale gravity models, Nucl. Phys. B 818 (2009) 28 [arXiv:0902.4894] [INSPIRE].

[85] N. Agarwal, V. Ravindran, V. Tiwari and A. Tripathi, Z boson pair production at the LHC to $O\left(\alpha_{s}\right)$ in TeV scale gravity models, Nucl. Phys. B 830 (2010) 248 [arXiv:0909.2651] [INSPIRE].

[86] N. Agarwal, V. Ravindran, V.K. Tiwari and A. Tripathi, Next-to-leading order QCD corrections to the $Z$ boson pair production at the LHC in Randall Sundrum model, Phys. Lett. B 686 (2010) 244 [arXiv: 0910.1551] [INSPIRE].

[87] N. Agarwal, V. Ravindran, V.K. Tiwari and A. Tripathi, Next-to-leading order QCD corrections to $W^{+} W^{-}$production at the LHC in Randall Sundrum model, Phys. Lett. B 690 (2010) 390 [arXiv: 1003.5445] [INSPIRE].

[88] N. Agarwal, V. Ravindran, V.K. Tiwari and A. Tripathi, $W^{+} W^{-}$production in Large extra dimension model at next-to-leading order in QCD at the LHC, Phys. Rev. D 82 (2010) 036001 [arXiv: 1003.5450] [INSPIRE].

[89] R. Frederix et al., Diphoton production in the ADD model to NLO+parton shower accuracy at the LHC, JHEP 12 (2012) 102 [arXiv:1209.6527] [INSPIRE].

[90] C. Englert, M. Spannowsky and M. Takeuchi, Measuring Higgs CP and couplings with hadronic event shapes, JHEP 06 (2012) 108 [arXiv: 1203.5788] [INSPIRE].

[91] C. Englert, D. Goncalves, G. Nail and M. Spannowsky, The shape of spins, Phys. Rev. D 88 (2013) 013016 [arXiv:1304.0033] [INSPIRE].

[92] R. Frederix et al., Scalar and pseudoscalar Higgs production in association with a top-antitop pair, Phys. Lett. B 701 (2011) 427 [arXiv:1104.5613] [INSPIRE].

[93] J. Pumplin et al., New generation of parton distributions with uncertainties from global QCD analysis, JHEP 07 (2002) 012 [hep-ph/0201195] [INSPIRE].

[94] A. Martin, W. Stirling, R. Thorne and G. Watt, Parton distributions for the LHC, Eur. Phys. J. C 63 (2009) 189 [arXiv:0901.0002] [InSPIRE].

[95] CTEQ collaboration, H. Lai et al., Global QCD analysis of parton structure of the nucleon: CTEQ5 parton distributions, Eur. Phys. J. C 12 (2000) 375 [hep-ph/9903282] [INSPIRE].

[96] M. Cacciari, G.P. Salam and G. Soyez, The Anti-k(t) jet clustering algorithm, JHEP 04 (2008) 063 [arXiv: 0802.1189] [INSPIRE].

[97] M. Cacciari, G.P. Salam and G. Soyez, FastJet User Manual, Eur. Phys. J. C 72 (2012) 1896 [arXiv: 1111.6097] [INSPIRE].

[98] S. Choi, D. Miller, M. Muhlleitner and P. Zerwas, Identifying the Higgs spin and parity in decays to Z pairs, Phys. Lett. B 553 (2003) 61 [hep-ph/0210077] [INSPIRE].

[99] D0 collaboration, V.M. Abazov et al., Search for Randall-Sundrum gravitons in the dielectron and diphoton final states with $5.4 \mathrm{fb}^{-1}$ of data from $p \bar{p}$ collisions at $\sqrt{s}=1.96 \mathrm{TeV}$, Phys. Rev. Lett. 104 (2010) 241802 [arXiv:1004.1826] [INSPIRE].

[100] CDF collaboration, Search for Randall-Sundrum Gravitons in the Diphoton Channel at CDF, Phys. Rev. D 83 (2011) 011102 [arXiv:1012.2795] [InSPIRE]. 
[101] CDF collaboration, Search for New Dielectron Resonances and Randall-Sundrum Gravitons at the Collider Detector at Fermilab, Phys. Rev. Lett. 107 (2011) 051801 [arXiv:1103.4650] [INSPIRE].

[102] J. Ellis, V. Sanz and T. You, Prima Facie Evidence against Spin-Two Higgs Impostors, Phys. Lett. B 726 (2013) 244 [arXiv:1211.3068] [INSPIRE].

[103] J. Ellis, V. Sanz and T. You, Associated Production Evidence against Higgs Impostors and Anomalous Couplings, Eur. Phys. J. C 73 (2013) 2507 [arXiv:1303.0208] [INSPIRE].

[104] P. Mathews, V. Ravindran, K. Sridhar and W. van Neerven, Next-to-leading order QCD corrections to the Drell-Yan cross section in models of TeV-scale gravity, Nucl. Phys. B 713 (2005) 333 [hep-ph/0411018] [INSPIRE].

[105] S. Karg, M. Krämer, Q. Li and D. Zeppenfeld, NLO QCD corrections to graviton production at hadron colliders, Phys. Rev. D 81 (2010) 094036 [arXiv:0911.5095] [InSPIRE].

[106] E.A. Mirabelli, M. Perelstein and M.E. Peskin, Collider signatures of new large space dimensions, Phys. Rev. Lett. 82 (1999) 2236 [hep-ph/9811337] [INSPIRE].

[107] P. Artoisenet, V. Lemaitre, F. Maltoni and O. Mattelaer, Automation of the matrix element reweighting method, JHEP 12 (2010) 068 [arXiv:1007.3300] [INSPIRE].

[108] K. Kondo, Dynamical Likelihood Method for Reconstruction of Events With Missing Momentum. 1: Method and Toy Models, J. Phys. Soc. Jap. 57 (1988) 4126 [inSPIRE].

[109] J.S. Gainer, K. Kumar, I. Low and R. Vega-Morales, Improving the sensitivity of Higgs boson searches in the golden channel, JHEP 11 (2011) 027 [arXiv:1108.2274] [INSPIRE].

[110] D. Stolarski and R. Vega-Morales, Directly Measuring the Tensor Structure of the Scalar Coupling to Gauge Bosons, Phys. Rev. D 86 (2012) 117504 [arXiv:1208.4840] [InSPIRE].

[111] A. Alves, Is the New Resonance Spin 0 or 2? Taking a Step Forward in the Higgs Boson Discovery, Phys. Rev. D 86 (2012) 113010 [arXiv:1209.1037] [INSPIRE].

[112] P. Avery et al., Precision studies of the Higgs boson decay channel $H \rightarrow Z Z \rightarrow 4 l$ with MEKD, Phys. Rev. D 87 (2013) 055006 [arXiv:1210.0896] [InSPIRE].

[113] CMS collaboration, Properties of the Higgs-like boson in the decay $H \rightarrow Z Z \rightarrow 4 l$ in $p p$ collisions at $\sqrt{s}=7$ and 8 TeV, CMS-PAS-HIG-13-002 (2013).

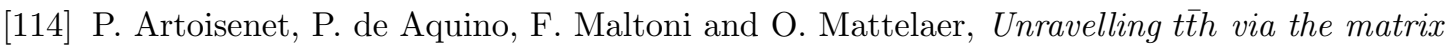
element method, arXiv:1304.6414 [INSPIRE].

[115] R. Frederix and S. Frixione, Merging meets matching in MC@NLO, JHEP 12 (2012) 061 [arXiv:1209.6215] [INSPIRE].

[116] J.P. Ralston, The Need to Fairly Confront Spin-1 for the New Higgs-like Particle, arXiv: 1211.2288 [INSPIRE].

[117] B.A. Kniehl and J.H. Kuhn, QCD Corrections to the Z Decay Rate, Nucl. Phys. B 329 (1990) 547 [INSPIRE]. 